\title{
Noether's second theorem for BRST symmetries
}

\author{
D.BASHKIROV ${ }^{1}$ \\ Department of Theoretical Physics, Moscow State University, 117234 Moscow, Russia \\ G.Giachetta ${ }^{2}$ \\ Department of Mathematics and Informatics, University of Camerino, 62032 Camerino \\ $(\mathrm{MC})$, Italy \\ L.MANGIAROTTI ${ }^{3}$ \\ Department of Mathematics and Informatics, University of Camerino, 62032 Camerino \\ $(\mathrm{MC})$, Italy \\ G. Sardanashvili ${ }^{4}$ \\ Department of Theoretical Physics, Moscow State University, 117234 Moscow, Russia
}

We present Noether's second theorem for graded Lagrangian systems of even and odd variables on an arbitrary body manifold $X$ in a general case of BRST symmetries depending on derivatives of dynamic variables and ghosts of any finite order. As a preliminary step, Noether's second theorem for Lagrangian systems on fiber bundles $Y \rightarrow X$ possessing gauge symmetries depending on derivatives of dynamic variables and parameters of arbitrary order is proved.

\section{INTRODUCTION}

Different variants of Noether's second theorem state that, if a Lagrangian admits symmetries depending on parameters, its variational derivatives obey certain relations, called the Noether identities. We present Noether's second theorem in the case of BRST transformations depending on derivatives (jets) of dynamic variables and ghosts of arbitrary order. In particular, this is the case of the field-antifield BRST theory and BV quantization. ${ }^{1,2}$ A special attention is paid to global aspects of Noether's second theorem as a preliminary step of the global analysis of BV quantization..$^{3,4}$

\footnotetext{
${ }^{1}$ Electronic mail: bashkir@phys.msu.ru

${ }^{2}$ Electronic mail: giovanni.giachetta@unicam.it

${ }^{3}$ Electronic mail: luigi.mangiarotti@unicam.it

${ }^{4}$ Electronic mail: sard@grav.phys.msu.su
} 
We start with a classical Lagrangian system on a fiber bundle $Y \rightarrow X$ subject to even gauge transformations depending on even dynamic variables, even parameters and their derivatives of any order. For this purpose, we consider Lagrangian formalism on the composite fiber bundle $E \rightarrow Y \rightarrow X$, where $E \rightarrow Y$ is a vector bundle of gauge parameters. Accordingly, gauge transformations are represented by a linear differential operator $v$ on $E$ taking its values in the vertical tangent bundle $V Y$ of $Y \rightarrow X$ (Section II). The Noether identity for a Lagrangian $L$ is defined as a differential operator $\Delta$ on the fiber bundle (13) which takes its values in the density-dual

$$
E^{*} \underset{Y}{\otimes} \wedge T^{*} X, \quad n=\operatorname{dim} X
$$

of $E$ and whose kernel contains the image of the Euler-Lagrange operator $\delta L$ of $L$, i.e., $\Delta \circ \delta L=0$ (Definition 4). Expressed in these terms, Noether's second theorem (Section III, Theorem 5) follows at once fom the properties of differential operators on dual fiber bundles (Appendix A, Theorem 16). Namely, there exists the intertwining operator $\eta(v)=\Delta$, $\eta(\Delta)=v$ such that

$$
\begin{aligned}
& \eta(\eta(v))=v, \quad \eta(\eta(\Delta))=\Delta, \\
& \eta\left(v \circ v^{\prime}\right)=\eta\left(v^{\prime}\right) \circ \eta(v), \quad \eta\left(\Delta^{\prime} \circ \Delta\right)=\eta(\Delta) \circ \eta\left(\Delta^{\prime}\right) .
\end{aligned}
$$

The appropriate notions of reducible Noether identities and gauge symmetries are formulated, and their equivalence with respect to the intertwining operator $\eta$ is proved (Section IV).

This formulation of Noether's second theorem is generalized to the case of graded Lagrangian systems of even and odd variables and BRST symmetries (Section VII, Theorem 15). We describe odd variables and their jets on an arbitrary smooth manifold $X$ as generating elements of the structure ring of a graded manifold whose body is $X .{ }^{4,5}$ This definition differs from that of jets of a graded fiber bundle, ${ }^{6}$ but reproduces the heuristic notion of jets of ghosts in the above mentioned field-antifield BRST theory. ${ }^{1,7}$

We consider BRST symmetries of a graded Lagrangian, i.e., its nilpotent odd symmetries depending on ghosts as parameters (Section VI). In particular, BRST symmetries come from the above mentioned gauge symmetries by replacement of even parameters with odd ghosts (Example 5). In this case, the nilpotency condition implies that original gauge symmetries form an algebra.

The key point is that, in order to define the Noether identity associated to BRST symmetries, one should introduce antifields and the Koszul-Tate differential. If a Noether identity is reducible, $(0 \leq k)$-stage ghosts and antighosts are called into play (Section VIII), 
and we come to the complete tuple of fields, ghosts and antifields in the field-antifield BRST theory. ${ }^{1}$ We however leave this theory outside the scope of the present work, and keep an original graded Lagrangian independent of ghosts and antifields.

\section{GAUGE SYSTEMS ON FIBER BUNDLES}

Recall that an $r$-order Lagrangian on a fiber bundle $Y \rightarrow X$ is defined as a density

$$
L=\mathcal{L} \omega: J^{r} Y \rightarrow \wedge \wedge^{n} T^{*} X, \quad \omega=d x^{1} \wedge \cdots \wedge d x^{n}
$$

on the $r$-order jet manifold $J^{r} Y$ of sections of $Y \rightarrow X$. Jet manifolds of $Y \rightarrow X$ make up the inverse system

$$
X \stackrel{\pi}{\longleftarrow} Y \stackrel{\pi_{0}^{1}}{\longleftarrow} J^{1} Y \longleftarrow \cdots J^{r-1} Y \stackrel{\pi_{r-1}^{r}}{\longleftarrow} J^{r} Y \longleftarrow \cdots
$$

In the sequel, the index $r=0$ stands for $Y$. Accordingly, we have the direct system

$$
\mathcal{O}^{*} X \stackrel{\pi^{*}}{\longrightarrow} \mathcal{O}^{*} Y \stackrel{\pi_{0}^{1 *}}{\longrightarrow} \mathcal{O}_{1}^{*} Y \longrightarrow \cdots \mathcal{O}_{r-1}^{*} Y \stackrel{\pi_{r-1}^{r}}{\longrightarrow} \mathcal{O}_{r}^{*} Y \longrightarrow \cdots
$$

of graded differential algebras (henceforth GDAs) $\mathcal{O}_{r}^{*} Y$ of exterior forms on jet manifolds $J^{r} Y$ with respect to the pull-back monomorphisms $\pi_{r-1}^{r}{ }^{*}$. Its direct limit $\mathcal{O}_{\infty}^{*} Y$ is a GDA consisting of all exterior forms on finite order jet manifolds modulo the pull-back identification.

The projective limit $\left(J^{\infty} Y, \pi_{r}^{\infty}: J^{\infty} Y \rightarrow J^{r} Y\right)$ of the inverse system (5) is a Fréchet manifold. ${ }^{8}$ A bundle atlas $\left\{\left(U_{Y} ; x^{\lambda}, y^{i}\right)\right\}$ of $Y \rightarrow X$ yields the coordinate atlas

$$
\left\{\left(\left(\pi_{0}^{\infty}\right)^{-1}\left(U_{Y}\right) ; x^{\lambda}, y_{\Lambda}^{i}\right)\right\}, \quad y_{\lambda+\Lambda}^{\prime i}=\frac{\partial x^{\mu}}{\partial x^{\prime \lambda}} d_{\mu} y_{\Lambda}^{\prime i}, \quad 0 \leq|\Lambda|
$$

of $J^{\infty} Y$, where $\Lambda=\left(\lambda_{k} \ldots \lambda_{1}\right)$ is a symmetric multi-index, $\lambda+\Lambda=\left(\lambda \lambda_{k} \ldots \lambda_{1}\right)$, and

$$
d_{\lambda}=\partial_{\lambda}+\sum_{0 \leq|\Lambda|} y_{\lambda+\Lambda}^{i} \partial_{i}^{\Lambda}, \quad d_{\Lambda}=d_{\lambda_{r}} \circ \cdots \circ d_{\lambda_{1}}
$$

are the total derivatives. There is the restriction epimorphism $\mathcal{O}_{\infty}^{*} Y \rightarrow \mathcal{O}_{\infty}^{*} U_{Y}$. Therefore, $\mathcal{O}_{\infty}^{*} Y$ can be written in a coordinate form where the horizontal one-forms $\left\{d x^{\lambda}\right\}$ and the contact one-forms $\left\{\theta_{\Lambda}^{i}=d y_{\Lambda}^{i}-y_{\lambda+\Lambda}^{i} d x^{\lambda}\right\}$ are generating elements of the $\mathcal{O}_{\infty}^{0} U_{Y^{-}}$algebra $\mathcal{O}_{\infty}^{*} U_{Y}$. Though $J^{\infty} Y$ is not a smooth manifold, elements of $\mathcal{O}_{\infty}^{*} Y$ are exterior forms on finite order jet manifolds and, therefore, their coordinate transformations are smooth.

There is the canonical decomposition $\mathcal{O}_{\infty}^{*} Y=\oplus \mathcal{O}_{\infty}^{k, m} Y$ of $\mathcal{O}_{\infty}^{*} Y$ into $\mathcal{O}_{\infty}^{0} Y$-modules $\mathcal{O}_{\infty}^{k, m} Y$ of $k$-contact and $m$-horizontal forms together with the corresponding projectors 
$h_{k}: \mathcal{O}_{\infty}^{*} Y \rightarrow \mathcal{O}_{\infty}^{k, *} Y$ and $h^{m}: \mathcal{O}_{\infty}^{*} Y \rightarrow \mathcal{O}_{\infty}^{*, m} Y$. Accordingly, the exterior differential on $\mathcal{O}_{\infty}^{*} Y$ is split into the sum $d=d_{H}+d_{V}$ of the nilpotent total and vertical differentials

$$
d_{H}(\phi)=d x^{\lambda} \wedge d_{\lambda} \phi, \quad d_{V}(\phi)=\theta_{\Lambda}^{i} \wedge \partial_{i}^{\Lambda} \phi, \quad \phi \in \mathcal{O}_{\infty}^{*} Y
$$

One also introduces the $\mathbb{R}$-module projector

$$
\left.\varrho=\sum_{0<k} \frac{1}{k} \bar{\varrho} \circ h_{k} \circ h^{n}, \quad \bar{\varrho}(\phi)=\sum_{0 \leq|\Lambda|}(-1)^{|\Lambda|} \theta^{i} \wedge\left[d_{\Lambda}\left(\partial_{i}^{\Lambda}\right\rfloor \phi\right)\right], \quad \phi \in \mathcal{O}_{\infty}^{>0, n} Y,
$$

of $\mathcal{O}_{\infty}^{*} Y$ such that $\varrho \circ d_{H}=0$, and the nilpotent variational operator $\delta=\varrho \circ d$ on $\mathcal{O}_{\infty}^{*, n} Y$. Let us put $\mathbf{E}_{k}=\varrho\left(\mathcal{O}_{\infty}^{k, n} Y\right)$. Then the GDA $\mathcal{O}_{\infty}^{*} Y$ is split into the well-known variational bicomplex. ${ }^{4,8-10}$ Here, we are concerned with its variational subcomplex

$$
0 \rightarrow \mathbb{R} \rightarrow \mathcal{O}_{\infty}^{0} Y \stackrel{d_{H}}{\longrightarrow} \mathcal{O}_{\infty}^{0,1} Y \cdots \stackrel{d_{H}}{\longrightarrow} \mathcal{O}_{\infty}^{0, n} Y \stackrel{\delta}{\longrightarrow} \mathbf{E}_{1} \stackrel{\delta}{\longrightarrow} \mathbf{E}_{2} \longrightarrow \cdots
$$

and the subcomplex of one-contact forms

$$
0 \rightarrow \mathcal{O}_{\infty}^{1,0} Y \stackrel{d_{H}}{\longrightarrow} \mathcal{O}_{\infty}^{1,1} Y \cdots \stackrel{d_{H}}{\longrightarrow} \mathcal{O}_{\infty}^{1, n} Y \stackrel{\varrho}{\longrightarrow} \mathbf{E}_{1} \rightarrow 0
$$

They possess the following cohomology. ${ }^{3,4,11}$

Theorem 1: The cohomology of the variational complex (10) equals the de Rham cohomology of $Y$.

Theorem 2: The complex (11) is exact.

Any finite order Lagrangian $L(4)$ is an element of $\mathcal{O}_{\infty}^{0, n} Y$, while

$$
\delta L=\mathcal{E}_{i} \theta^{i} \wedge \omega=\sum_{0 \leq|\Lambda|}(-1)^{|\Lambda|} d_{\Lambda}\left(\partial_{i}^{\Lambda} \mathcal{L}\right) \theta^{i} \wedge \omega \in \mathbf{E}_{1}
$$

is its Euler-Lagrange operator taking the values in the vector bundle

$$
T^{*} Y \underset{Y}{\wedge}\left(\stackrel{n}{\wedge} T^{*} X\right)=V^{*} Y \underset{Y}{\otimes} \stackrel{n}{\wedge} T^{*} X
$$

The components $\mathcal{E}_{i}$ of $\delta L$ are called the variational derivatives. We further abbreviate $A \approx 0$ with an equality which holds on-shell. This means that $A$ is an element of a module over the ideal $I_{L}$ of the ring $\mathcal{O}_{\infty}^{0} Y$ which is locally generated by the variational derivatives $\mathcal{E}_{i}$ (12) and their total derivatives $d_{\Lambda} \mathcal{E}_{i}$. Thus, $I_{L}$ is a differential ideal.

By virtue of Theorem 1, every $\delta$-closed Lagrangian $L \in \mathcal{O}_{\infty}^{0, n} Y$ is the sum

$$
L=h_{0} \psi+d_{H} \sigma, \quad \sigma \in \mathcal{O}_{\infty}^{0, n-1} Y,
$$


where $\psi$ is a closed $n$-form on $Y$. Theorem 2 provides the $\mathbb{R}$-module decomposition

$$
\mathcal{O}_{\infty}^{1, n} Y=\mathbf{E}_{1} \oplus d_{H}\left(\mathcal{O}_{\infty}^{1, n-1} Y\right)
$$

Given a Lagrangian $L \in \mathcal{O}_{\infty}^{0, n} Y$, we have the corresponding decomposition

$$
d L=\delta L-d_{H} \Xi
$$

where $\Xi_{L}=\Xi+L$ is a Lepagean equivalent of $L$.

Let $\mathfrak{d} \mathcal{O}_{\infty}^{0} Y$ be the $\mathcal{O}_{\infty}^{0} Y$-module of derivations of the $\mathbb{R}$-ring $\mathcal{O}_{\infty}^{0} Y$. Any $\vartheta \in \mathfrak{d} \mathcal{O}_{\infty}^{0} Y$ yields the graded derivation (the interior product) $\vartheta\rfloor \phi$ of the GDA $\mathcal{O}_{\infty}^{*} Y$ given by the relations

$$
\begin{aligned}
& \vartheta\rfloor d f=\vartheta(f), \quad f \in \mathcal{O}_{\infty}^{0} Y, \\
& \left.\vartheta\rfloor(\phi \wedge \sigma)=(\vartheta\rfloor \phi) \wedge \sigma+(-1)^{|\phi|} \phi \wedge(\vartheta\rfloor \sigma\right), \quad \phi, \sigma \in \mathcal{O}_{\infty}^{*} Y,
\end{aligned}
$$

and its derivation (the Lie derivative)

$$
\begin{aligned}
& \left.\left.\mathbf{L}_{\vartheta} \phi=\vartheta\right\rfloor d \phi+d(\vartheta\rfloor \phi\right), \quad \phi \in \mathcal{O}_{\infty}^{*} Y, \\
& \mathbf{L}_{\vartheta}\left(\phi \wedge \phi^{\prime}\right)=\mathbf{L}_{\vartheta}(\phi) \wedge \phi^{\prime}+\phi \wedge \mathbf{L}_{\vartheta}\left(\phi^{\prime}\right) .
\end{aligned}
$$

Relative to an atlas $(7)$, a derivation $\vartheta \in \mathfrak{d O}_{\infty}^{0} \operatorname{reads}^{4}$

$$
\vartheta=\vartheta^{\lambda} \partial_{\lambda}+\vartheta^{i} \partial_{i}+\sum_{|\Lambda|>0} \vartheta_{\Lambda}^{i} \partial_{i}^{\Lambda}
$$

where the tuple of derivations $\left\{\partial_{\lambda}, \partial_{i}^{\Lambda}\right\}$ is defined as the dual of the set $\left\{d x^{\lambda}, d y_{\Lambda}^{i}\right\}$ of generating elements for the $\mathcal{O}_{\infty}^{0}$-algebra $\mathcal{O}_{\infty}^{*}$ with respect to the interior product $\rfloor$, and local functions $\vartheta^{\lambda}, \vartheta^{i}, \vartheta_{\Lambda}^{i} \in \mathcal{O}_{\infty}^{0}$ obey the transformation law

$$
\vartheta^{\prime \lambda}=\frac{\partial x^{\prime \lambda}}{\partial x^{\mu}} \vartheta^{\mu}, \quad \vartheta_{\Lambda}^{i}=\sum_{|\Sigma| \leq|\Lambda|} \frac{\partial y_{\Lambda}^{\prime i}}{\partial y_{\Sigma}^{j}} \vartheta_{\Sigma}^{j}+\frac{\partial y_{\Lambda}^{\prime i}}{\partial x^{\mu}} v^{\mu} .
$$

Note that the tuple of derivations $\left\{\partial_{i}^{\Lambda}\right\}$ is the dual of the basis $\left\{\theta_{\Lambda}^{i}\right\}$ of contact forms.

A derivation $\vartheta$ is called contact if the Lie derivative $\mathbf{L}_{\vartheta}$ (16) preserves the contact ideal of the GDA $\mathcal{O}_{\infty}^{*} Y$ generated by contact forms. A derivation $\vartheta(17)$ is contact iff

$$
\vartheta_{\Lambda}^{i}=d_{\Lambda}\left(\vartheta^{i}-y_{\mu}^{i} \vartheta^{\mu}\right)+y_{\mu+\Lambda}^{i} \vartheta^{\mu}, \quad 0<|\Lambda|
$$

Any contact derivation admits the horizontal splitting

$$
\vartheta=\vartheta_{H}+\vartheta_{V}=\vartheta^{\lambda} d_{\lambda}+\left(v^{i} \partial_{i}+\sum_{0<|\Lambda|} d_{\Lambda} v^{i} \partial_{i}^{\Lambda}\right), \quad v^{i}=\vartheta^{i}-y_{\mu}^{i} \vartheta^{\mu}
$$


relative to the canonical connection $\nabla=d x^{\lambda} \otimes d_{\lambda}$ on the $C^{\infty}(X)$-ring $\mathcal{O}_{\infty}^{0}{ }^{5,12}$ Its vertical part $\vartheta_{V}$ is completely determined by the first summand

$$
v=v^{i}\left(x^{\lambda}, y_{\Lambda}^{i}\right) \partial_{i}, \quad 0 \leq|\Lambda| \leq k .
$$

This is a section of the pull-back $V Y \underset{Y}{\times} J^{k} Y \rightarrow J^{k} Y$ of the vertical tangent bundle $V Y \rightarrow Y$ onto $J^{k} Y{ }^{13}$ i.e., $v(21)$ is a $k$-order $V Y$-valued differential operator on $Y$ (see Appendix A). One calls this differential operator the generalized vector field on $Y$.

Proposition 3: It follows from the splitting (15) that the Lie derivative of a Lagrangian $L$ (4) along a contact derivation $\vartheta(20)$ fulfills the first variational formula

$$
\left.\left.\left.\mathbf{L}_{\vartheta} L=v\right\rfloor \delta L+d_{H}\left(h_{0}(\vartheta\rfloor \Xi_{L}\right)\right)+\mathcal{L} d_{V}\left(\vartheta_{H}\right\rfloor \omega\right),
$$

where $\Xi_{L}$ is a Lepagean equivalent of $L .^{4}$

A contact derivation $\vartheta(20)$ is called variational if the Lie derivative $(22)$ is $d_{H}$-exact, i.e., $\mathbf{L}_{\vartheta} L=d_{H} \sigma, \sigma \in \mathcal{O}_{\infty}^{0, n-1}$. A glance at the expression (22) shows that: (i) a contact derivation $\vartheta$ is variational only if it is projected onto $X$ (i.e., its components $\vartheta^{\lambda}$ depend only on coordinates on $X$ ), (ii) $\vartheta$ is variational iff its vertical part $\vartheta_{V}$ is variational, (iii) it is variational if $v\rfloor \delta L$ is $d_{H}$-exact.

By virtue of item (ii), we can restrict our consideration to vertical contact derivations $\vartheta=\vartheta_{V}$. A generalized vector field $v(21)$ is called a variational symmetry of a Lagrangian $L$ if it generates a variational vertical contact derivation.

One can also consider locally-variational contact derivations when the Lie derivative (22) is $\delta$-closed, but any locally-variational gauge symmetry is always variational (see Remark 1 below).

Turn now to the notion of a gauge symmetry. A Lagrangian system on a fiber bundle $Y \rightarrow X$ is said to be a gauge theory if its Lagrangian $L$ admits a family of variational symmetries parameterized by elements of a vector bundle $E \rightarrow Y$ as follows.

Let $E \rightarrow Y$ be a vector bundle coordinated by $\left(x^{\lambda}, y^{i}, \xi^{r}\right)$. Given a Lagrangian $L$ on $Y$, let us consider its pull-back, say again $L$, onto $E$. Let $\vartheta_{E}$ be a vertical contact derivation of the $\mathbb{R}$-ring $\mathcal{O}_{\infty}^{0} E$ whose restriction

$$
\vartheta=\left.\vartheta_{E}\right|_{\mathcal{O}_{\infty}^{0} Y}=\sum_{0 \leq|\Lambda|} d_{\Lambda} v^{i} \partial_{i}^{\Lambda}
$$

to $\mathcal{O}_{\infty}^{0} Y \subset \mathcal{O}_{\infty}^{0} E$ is linear in coordinates $\xi_{\Xi}^{r}$. It is determined by a generalized vector field (i.e., a $V E$-valued differential operator) $v_{E}$ on $E$ whose canonical projection

$$
v: J^{k} E \stackrel{v_{E}}{\longrightarrow} V E \rightarrow \underset{Y}{\times} \underset{Y}{\times} V
$$


(see the exact sequence (35) below) is a linear $V Y$-valued differential operator

$$
v=\sum_{0 \leq|\Xi| \leq m} v_{r}^{i, \Xi}\left(x^{\lambda}, y_{\Sigma}^{i}\right) \xi_{\Xi}^{r} \partial_{i}
$$

on $E$. Let $\vartheta_{E}$ be a variational symmetry of a Lagrangian $L$ on $E$, i.e.,

$$
\left.\left.v_{E}\right\rfloor \delta L=v\right\rfloor \delta L=d_{H} \sigma
$$

Then one says that $v(24)$ is a gauge symmetry of a Lagrangian $L$ on $Y$.

Remark 1: As was mentioned above, any locally-variational gauge symmetry $\vartheta_{E}$, when the Lie derivative $\mathbf{L}_{\vartheta_{E}} L$ is $\delta$-closed, is variational. By virtue of Theorem $1, \mathbf{L}_{\vartheta_{E}} L$ takes the form (14) where $\psi$ is a closed form on $E$. Since $E \rightarrow Y$ is a vector bundle, $Y$ is a strong deformation retract of $E$ and, consequently, the de Rham cohomology of $E$ equals that of $Y$. Then any closed form on $E$ is the sum of the pull-back of a closed form on $Y$ and an exact form on $E$. The former is independent of fiber coordinates $\xi^{r}$ on $E \rightarrow Y$. Since the Lie derivative $\mathbf{L}_{\vartheta} L$ is linear in $\xi_{\Lambda}^{r}$, it is always $d_{H}$-exact, i.e., $\vartheta_{E}$ is variational.

\section{NOETHER'S SECOND THEOREM I}

Let us start with the notion of the Noether identity.

Definition 4: Given a Lagrangian $L(4)$ and its Euler-Lagrange operator $\delta L$ (12), let $E \rightarrow Y$ be a vector bundle and $\Delta$ a linear differential operator of order $0 \leq m$ on the vector bundle (13) with the values in the density-dual $\bar{E}^{*}(1)$ of $E$ such that

$$
\Delta \circ \delta L=0
$$

This condition is called the Noether identity, and $\Delta$ is the Noether operator.

Given bundle coordinates $\left(x^{\lambda}, y^{i}, \bar{y}_{i}\right)$ on the fiber bundle $(13)$ and $\left(x^{\lambda}, y^{i}, \xi^{r}\right)$ on $E$, a Noether operator $\Delta$ in Definition 4 is represented by the density

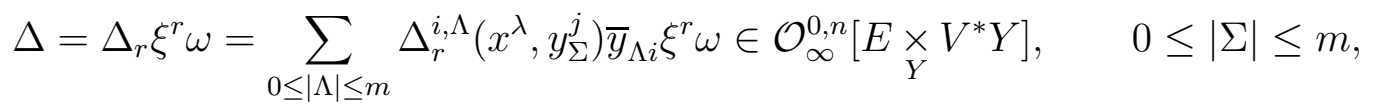

(see Appendix A). Then the Noether identity (26) takes the coordinate form

$$
\left[\sum_{0 \leq|\Lambda| \leq m} \Delta_{r}^{i, \Lambda} d_{\Lambda} \mathcal{E}_{i}\right] \xi^{r} \omega=0
$$


where $\mathcal{E}_{i}$ are variational derivatives (12).

Remark 2: We further use the relations

$$
\begin{aligned}
& \sum_{0 \leq|\Lambda| \leq k} B^{\Lambda} d_{\Lambda} A^{\prime}=\sum_{0 \leq|\Lambda| \leq k}(-1)^{|\Lambda|} d_{\Lambda}\left(B^{\Lambda}\right) A^{\prime}+d_{H} \sigma \\
& \sum_{0 \leq|\Lambda| \leq k}(-1)^{|\Lambda|} d_{\Lambda}\left(B^{\Lambda} A\right)=\sum_{0 \leq|\Lambda| \leq k} \eta(B)^{\Lambda} d_{\Lambda} A, \\
& \eta(B)^{\Lambda}=\sum_{0 \leq|\Sigma| \leq k-|\Lambda|}(-1)^{|\Sigma+\Lambda|} C_{|\Sigma+\Lambda|}^{|\Sigma|} d_{\Sigma} B^{\Sigma+\Lambda}, \quad C_{b}^{a}=\frac{b !}{a !(b-a) !} \\
& (\eta \circ \eta)(B)^{\Lambda}=B^{\Lambda}
\end{aligned}
$$

for any exterior forms $A^{\prime} \in \mathcal{O}_{\infty}^{*, n} Z, A \in \mathcal{O}_{\infty}^{*} Z$ and any local function $B^{\Lambda} \in \mathcal{O}_{\infty}^{0} Z$ on jet manifolds of a fiber bundle $Z \rightarrow X$.

Theorem 5: If a Lagrangian $L(4)$ admits a gauge symmetry $v(24)$, its Euler-Lagrange operator obeys the Noether identity (28) where

$$
\Delta_{r}^{i, \Lambda}=\eta(v)_{r}^{i, \Lambda}=\sum_{0 \leq|\Sigma| \leq m-|\Lambda|}(-1)^{|\Sigma+\Lambda|} C_{|\Sigma+\Lambda|}^{|\Sigma|} d_{\Sigma} v_{r}^{i, \Sigma+\Lambda}
$$

Conversely, if the Euler-Lagrange operator of a Lagrangian $L$ obeys the Noether identity (28), this Lagrangian admits a gauge symmetry $v(24)$ where

$$
v_{r}^{i, \Lambda}=\eta(\Delta)_{r}^{i, \Lambda}=\sum_{0 \leq|\Sigma| \leq m-|\Lambda|}(-1)^{|\Sigma+\Lambda|} C_{|\Sigma+\Lambda|}^{|\Sigma|} d_{\Sigma} \Delta_{r}^{i, \Sigma+\Lambda}
$$

The relations (2) hold.

Proof: Given a differential operator $v(24)$, the operator $\Delta=\eta(v)$ expressed in the coordinate form (33) is defined in accordance with Theorem 16. Since the density

$$
v\rfloor \delta L=v^{i} \mathcal{E}_{i} \omega=\sum_{0 \leq|\Xi| \leq m} v_{r}^{i, \Xi} \xi_{\Xi}^{r} \mathcal{E}_{i} \omega
$$

is $d_{H}$-exact, the Noether identity

$$
\delta(v\rfloor \delta L)=\eta(v) \circ \delta L=0
$$

holds. Conversely, any Noether operator $\Delta(27)$ defines the $V Y$-valued differential operator $v=\eta(\Delta)$ on $E$ expressed in the coordinate form (34). This differential operator gives rise to a $V E$-valued differential operator (i.e., a generalized vector field) $v_{E}$ on $E$ and, thus, 
defines a contact derivation $\vartheta_{E}$ of $\mathcal{O}_{\infty}^{0} E$. Indeed, let us consider the exact sequence of vector bundles

$$
0 \rightarrow V_{Y} E \rightarrow V E \rightarrow \underset{Y}{E} \times Y Y \rightarrow 0,
$$

where $V_{Y} E$ is the vertical tangent bundle of $E \rightarrow Y$. Any splitting $\Gamma$ of this exact sequence lifts $v$ to the generalized vector field $v_{E}=\Gamma \circ v$ on $E$, but the Lie derivative $\mathbf{L}_{\vartheta_{E}} L$ is independent of the choice of a splitting $\Gamma$. Due to the Noether identity (28), we obtain

$$
\begin{aligned}
0= & \sum_{0 \leq|\Lambda| \leq m} \xi^{r} \Delta_{r}^{i, \Lambda} d_{\Lambda} \mathcal{E}_{i} \omega=\sum_{0 \leq|\Lambda| \leq m}(-1)^{|\Lambda|} d_{\Lambda}\left(\xi^{r} \Delta_{r}^{i, \Lambda}\right) \mathcal{E}_{i} \omega+d_{H} \sigma= \\
& \left.\sum_{0 \leq|\Xi| \leq m} v_{r}^{i, \Xi} \xi_{\Xi}^{r} \mathcal{E}_{i} \omega+d_{H} \sigma=v\right] \delta L+d_{H} \sigma,
\end{aligned}
$$

i.e., $v$ is a gauge symmetry of $L$. Due to the equality (32), the relations (2) hold.

Example 3: If a gauge symmetry

$$
v=\left(v_{r}^{i} \xi^{r}+v_{r}^{i, \mu} \xi_{\mu}^{r}\right) \partial_{i}
$$

is of first jet order in parameters, the corresponding Noether operator and Noether identity read

$$
\begin{aligned}
& \Delta_{r}^{i}=v_{r}^{i}-d_{\mu} v_{r}^{i, \mu}, \quad \Delta_{r}^{i, \mu}=-v_{r}^{i, \mu} \\
& {\left[v_{r}^{i} \mathcal{E}_{i}-d_{\mu}\left(v_{r}^{i, \mu} \mathcal{E}_{i}\right)\right] \xi^{r} \omega=0}
\end{aligned}
$$

Any Lagrangian $L$ has gauge symmetries. In particular, there always exist trivial gauge symmetries

$$
v=\sum_{\Lambda} \eta(M)_{r}^{i, \Lambda} \xi_{\Lambda}^{r}, \quad M_{r}^{i, \Lambda}=\sum_{\Sigma} T^{i, j, \Lambda, \Sigma} d_{\Sigma} \mathcal{E}_{j}, \quad T_{r}^{j, i, \Lambda, \Sigma}=-T_{r}^{i, j, \Sigma, \Lambda},
$$

corresponding to the trivial Noether identity

$$
\sum_{\Sigma, \Lambda} T_{r}^{j, i, \Lambda, \Sigma} d_{\Sigma} \mathcal{E}_{j} d_{\Lambda} \mathcal{E}_{i}=0
$$

Furthermore, given a gauge symmetry $v(24)$, let $h$ be a linear differential operator on some vector bundle $E^{\prime} \rightarrow Y$, coordinated by $\left(x^{\lambda}, y^{i}, \xi^{\prime s}\right)$, with values in the vector bundle $E$. Then the composition

$$
v_{0}^{\prime}=v \circ h=v_{s}^{\prime i, \Lambda} \xi_{\Lambda}^{\prime s} \partial_{i}, \quad v_{s}^{\prime i, \Lambda}=\sum_{\Xi+\Xi^{\prime}=\Lambda} \sum_{0 \leq|\Sigma| \leq m-|\Xi|} v_{r}^{i, \Xi+\Sigma} d_{\Sigma} h_{s}^{r, \Xi^{\prime}},
$$


is a variational symmetry of the pull-back onto $E^{\prime}$ of a Lagrangian $L$ on $Y$, i.e., a gauge symmetry of $L$. In view of this ambiguity, we agree to say that a gauge symmetry $v(24)$ of a Lagrangian $L$ is complete if a different gauge symmetry $v_{0}^{\prime}$ of $L$ factors through $v$ as

$$
v_{0}^{\prime}=v \circ h+T, \quad T \approx 0
$$

A complete gauge symmetry always exists, but the vector bundle of its parameters need not be finite-dimensional.

Accordingly, given the Noether operator (27), let $H$ be a linear differential operator on $\bar{E}^{*}$ with values in the density-dual $\bar{E}^{*}(1)$ of some vector bundle $E^{\prime} \rightarrow Y$. Then the composition $\Delta^{\prime}=H \circ \Delta$ ia also a Noether operator. We agree to call the Noether operator (27) complete if a different Noether operator $\Delta^{\prime}$ factors through $\Delta$ as

$$
\Delta^{\prime}=H \circ \Delta+F, \quad F \approx 0 .
$$

Proposition 6: A gauge symmetry $v$ of a Lagrangian $L$ is complete iff so is the associated Noether operator.

Proof: The proof follows at once from Proposition 17 in Appendix A. Given a gauge symmetry $v$ of $L$, let $v_{0}^{\prime}$ be a different gauge symmetry. If $\eta(v)$ is a complete Noether operator, then

$$
\eta\left(v_{0}^{\prime}\right)=H \circ \eta(v)+F, \quad F \approx 0
$$

and, by virtue of the relations (3), we have

$$
v_{0}^{\prime}=v \circ \eta(H)+\eta(F)
$$

where $\eta(F) \approx 0$ because $I_{L}$ is a differential ideal. The converse is similarly proved.

Example 4: Let us consider the gauge theory of principal connections on a principal bundle $P \rightarrow X$ with a structure Lie group $G{ }^{12}$ These connections are represented by sections of the quotient

$$
C=J^{1} P / G \rightarrow X
$$

called the bundle of principal connections. This is an affine bundle coordinated by $\left(x^{\lambda}, a_{\lambda}^{r}\right)$ such that, given a section $A$ of $C \rightarrow X$, its components $A_{\lambda}^{r}=a_{\lambda}^{r} \circ A$ are coefficients of the familiar local connection form (i.e., gauge potentials). Let $J^{\infty} C$ be the infinite order jet manifold of $C \rightarrow X$ coordinated by $\left(x^{\lambda}, a_{\Lambda \lambda}^{r}\right), 0 \leq|\Lambda|$. We consider the GDA $\mathcal{O}_{\infty}^{*} C$. 
Infinitesimal generators of local one-parameter groups of automorphisms of a principal bundle $P$ are $G$-invariant projectable vector fields on $P \rightarrow X$. They are associated to sections of the vector bundle $T_{G} P=T P / G \rightarrow X$. This bundle is endowed with the coordinates $\left(x^{\lambda}, \tau^{\lambda}=\dot{x}^{\lambda}, \xi^{r}\right)$ with respect to the fiber bases $\left\{\partial_{\lambda}, e_{r}\right\}$ for $T_{G} P$, where $\left\{e_{r}\right\}$ is the basis for the right Lie algebra $\mathfrak{g}$ of $G$ such that $\left[e_{p}, e_{q}\right]=c_{p q}^{r} e_{r}$. If

$$
u=u^{\lambda} \partial_{\lambda}+u^{r} e_{r}, \quad v=v^{\lambda} \partial_{\lambda}+v^{r} e_{r}
$$

are sections of $T_{G} P \rightarrow X$, their bracket reads

$$
[u, v]=\left(u^{\mu} \partial_{\mu} v^{\lambda}-v^{\mu} \partial_{\mu} u^{\lambda}\right) \partial_{\lambda}+\left(u^{\lambda} \partial_{\lambda} v^{r}-v^{\lambda} \partial_{\lambda} u^{r}+c_{p q}^{r} u^{p} v^{q}\right) e_{r}
$$

Any section $u$ of the vector bundle $T_{G} P \rightarrow X$ yields the vector field

$$
u_{C}=u^{\lambda} \partial_{\lambda}+\left(c_{p q}^{r} a_{\lambda}^{p} u^{q}+\partial_{\lambda} u^{r}-a_{\mu}^{r} \partial_{\lambda} u^{\mu}\right) \partial_{r}^{\lambda}
$$

on the bundle of principal connections $C$ (39). It is an infinitesimal generator of a oneparameter group of automorphisms of $C .{ }^{12}$ Let us consider the bundle product

$$
E=C \underset{X}{\times} T_{G} P
$$

coordinated by $\left(x^{\lambda}, \tau^{\lambda}, \xi^{r}, a_{\lambda}^{r}\right)$. It can be provided with the generalized vector field

$$
v_{E}=v=\left(c_{p q}^{r} a_{\lambda}^{p} \xi^{q}+\xi_{\lambda}^{r}-a_{\mu}^{r} \tau_{\lambda}^{\mu}-\tau^{\mu} a_{\mu \lambda}^{r}\right) \partial_{r}^{\lambda}
$$

For instance, this is a gauge symmetry of the global Chern-Simons Lagrangian. ${ }^{14}$ Let us consider a subbundle $V_{G} P=V P / G \rightarrow X$ of the vector bundle $T_{G} X$ coordinated by $\left(x^{\lambda}, \xi^{r}\right)$. Its sections $u=u^{r} e_{r}$ are infinitesimal generators of vertical automorphisms of $P$. Let us restrict the bundle product (43) to

$$
E=\underset{X}{\underset{X}{\times}} V_{G} P
$$

It is provided with the generalized vector field

$$
v_{E}=v=\left(c_{p q}^{r} a_{\lambda}^{p} \xi^{q}+\xi_{\lambda}^{r}\right) \partial_{r}^{\lambda}
$$

This is a gauge symmetry of the Yang-Mills Lagrangians, ${ }^{14}$ and yields the well-known Noether identity

$$
\left[c_{p q}^{r} a_{\lambda}^{p} \mathcal{E}_{r}^{\lambda}-d_{\lambda}\left(\mathcal{E}_{q}^{\lambda}\right)\right] \xi^{q} \omega=0
$$




\section{REDUCIBLE GAUGE THEORIES}

Recall that the notion of a reducible Noether identity has come from that of a reducible constraint, ${ }^{15}$ but it involves differential relations.

Definition 7: A complete Noether operator $\Delta \not \approx 0$ (27) and the corresponding Noether identity (26) are said to be $N$-stage reducible $(N=0,1, \ldots)$ if there exist vector bundles $E_{k} \rightarrow Y$ and differential operators $\Delta_{k}, k=0, \ldots, N$, such that:

(i) $\Delta_{k}$ is a linear differential operator on the density-dual $\bar{E}_{k-1}^{*}$ of $E_{k-1}$ with values in the density-dual $\bar{E}_{k}^{*}$ of $E_{k}$, where $E_{-1}=E$;

(ii) $\Delta_{k} \not \approx 0$ for all $k=0, \ldots, N$;

(iii) $\Delta_{k} \circ \Delta_{k-1} \approx 0$ for all $k=0, \ldots, N$, where $\Delta_{-1}=\Delta$;

(iv) if $\Delta_{k}^{\prime}$ is another differential operator possessing these properties, then it factors through $\Delta_{k}$ on-shell.

In particular, a zero-stage reducible Noether operator is called reducible. In this case, given bundle coordinates $\left(x^{\lambda}, y^{i}, \bar{\xi}_{r}\right)$ on $\bar{E}^{*}$ and $\left(x^{\lambda}, y^{i}, \xi^{r_{0}}\right)$ on $E_{0}$, a differential operator $\Delta_{0}$ reads

$$
\Delta_{0}=\sum_{0 \leq|\Xi| \leq m_{0}} \Delta_{r_{0}}^{r, \Xi} \bar{\xi}_{\Xi r} \xi^{r_{0}} \omega .
$$

Then the reduction condition $\Delta_{0} \circ \Delta \approx 0$ takes the coordinate form

$$
\sum_{0 \leq|\Xi| \leq m_{0}} \Delta_{r_{0}}^{r, \Xi} d_{\Xi}\left(\sum_{0 \leq|\Lambda| \leq m} \Delta_{r}^{i, \Lambda} \bar{y}_{\Lambda i}\right) \xi^{r_{0}} \omega \approx 0,
$$

i.e., the left hand-side of this expression takes the form

$$
\sum_{0 \leq|\Sigma| \leq m_{0}+m} M_{r_{0}}^{i, \Sigma} \bar{y}_{\Sigma i} \xi^{r_{0}} \omega
$$

where all the coefficients $M_{r_{0}}^{i, \Sigma}$ belong to the ideal $I_{L}$.

Definition 8: A complete gauge symmetry $v \not \approx 0$ (24) is said to be $N$-stage reducible if there exist vector bundles $E_{k}$ and differential operators $v^{k}, k=0, \ldots, N$, such that:

(i) $v^{k}$ is a linear differential operator on the vector bundle $E_{k}$ with values in the vector bundle $E_{k-1}$;

(ii) $v^{k} \not \approx 0$ for all $k=0, \ldots, N$;

(iii) $v^{k-1} \circ v^{k} \approx 0$ for all $k=0, \ldots, N$, where $v^{k}, k=-1$, stands for $v$; 
(iv) if $v^{\prime k}$ is another differential operator possessing these properties, then $v^{\prime k}$ factors through $v^{k}$ on-shell.

Theorem 9: A gauge symmetry $v$ is $N$-stage reducible iff so is the associated Noether identity.

Proof: The proof follows at once from Theorem 16 and Proposition 17. Let us put $\Delta_{k}=$ $\eta\left(v^{k}\right), k=0, \ldots, N$. If $v^{k} \approx 0$, then $\eta\left(v^{k}\right) \approx 0$ because $I_{L}$ is a differential ideal. By the same reason, if $v^{k-1}$ and $v^{k}$ obey the reduction condition $v^{k-1} \circ v^{k} \approx 0$, then

$$
\eta\left(v^{k-1} \circ v^{k}\right)=\eta\left(v^{k}\right) \circ \eta\left(v^{k-1}\right) \approx 0 .
$$

The converse is justified in the same way. The equivalence of the conditions in items (iv) of Definitions 7 and 8 is proved similarly to that in Proposition 6.

\section{GRADED LAGRANGIAN SYSTEMS}

Recall that, by virtue of Batchelor's theorem, ${ }^{16}$ any graded manifold $(X, \mathfrak{A})$ with a body $X$ is isomorphic to the one whose structure sheaf $\mathfrak{A}_{Q}$ is formed by germs of sections of the exterior product

$$
\wedge Q^{*}=\mathbb{R} \underset{X}{\oplus} Q^{*} \oplus_{X} \wedge^{2} Q^{*}{\underset{X}{\oplus}}_{\cdots} \cdots
$$

where $Q^{*}$ is the dual of some real vector bundle $Q \rightarrow X$ of fiber dimension $m$. In field models, a vector bundle $Q$ is usually given from the beginning. Therefore, we consider graded manifolds $\left(X, \mathfrak{A}_{Q}\right)$ where Batchelor's isomorphism is fixed, and call $\left(X, \mathfrak{A}_{Q}\right)$ the simple graded manifold constructed from $Q$. The structure ring $\mathcal{A}_{Q}$ of sections of $\mathfrak{A}_{Q}$ consists of sections of the exterior bundle (49) called graded functions. Given bundle coordinates $\left(x^{\lambda}, q^{a}\right)$ on $Q$ with transition functions $q^{a}=\rho_{b}^{a} q^{b}$, let $\left\{c^{a}\right\}$ be the corresponding fiber bases for $Q^{*} \rightarrow X$, together with transition functions $c^{\prime a}=\rho_{b}^{a} c^{b}$. Then $\left(x^{\lambda}, c^{a}\right)$ is called the local basis for the graded manifold $\left(X, \mathfrak{A}_{Q}\right)$. With respect to this basis, graded functions read

$$
f=\sum_{k=0}^{m} \frac{1}{k !} f_{a_{1} \ldots a_{k}} c^{a_{1}} \cdots c^{a_{k}},
$$

where $f_{a_{1} \cdots a_{k}}$ are local smooth real functions on $X$, and we omit the symbol of the exterior product of elements $c^{a}$. 
Given a graded manifold $\left(X, \mathfrak{A}_{Q}\right)$, let $\mathfrak{d} \mathcal{A}_{Q}$ be the $\mathcal{A}_{Q}$-module of $\mathbb{Z}_{2}$-graded derivations of the $\mathbb{Z}_{2}$-graded ring $\mathcal{A}_{Q}$, i.e.,

$$
u\left(f f^{\prime}\right)=u(f) f^{\prime}+(-1)^{[u][f]} f u\left(f^{\prime}\right), \quad u \in \mathfrak{d} \mathcal{A}_{Q}, \quad f, f^{\prime} \in \mathcal{A}_{Q},
$$

where [.] denotes the Grassmann parity. Its elements are called $\mathbb{Z}_{2}$-graded (or, simply, graded) vector fields on $\left(X, \mathfrak{A}_{Q}\right)$. Due to the canonical splitting $V Q=Q \times Q$, the vertical tangent bundle $V Q \rightarrow Q$ of $Q \rightarrow X$ can be provided with the fiber basis $\left\{\partial_{a}\right\}$ which is the dual of $\left\{c^{a}\right\}$. Then a graded vector field takes the local form $u=u^{\lambda} \partial_{\lambda}+u^{a} \partial_{a}$, where $u^{\lambda}, u^{a}$ are local graded functions. It acts on $\mathcal{A}_{Q}$ by the rule

$$
\left.u\left(f_{a \ldots b} c^{a} \cdots c^{b}\right)=u^{\lambda} \partial_{\lambda}\left(f_{a \ldots b}\right) c^{a} \cdots c^{b}+u^{d} f_{a \ldots b} \partial_{d}\right\rfloor\left(c^{a} \cdots c^{b}\right) .
$$

This rule implies the corresponding transformation law

$$
u^{\prime \lambda}=u^{\lambda}, \quad u^{\prime a}=\rho_{j}^{a} u^{j}+u^{\lambda} \partial_{\lambda}\left(\rho_{j}^{a}\right) c^{j} .
$$

Then one can show ${ }^{5,12}$ that graded vector fields on a simple graded manifold can be represented by sections of the vector bundle $\mathcal{V}_{Q} \rightarrow X$ which is locally isomorphic to the vector bundle $\wedge Q^{*} \otimes_{X}\left(Q \oplus_{X} T X\right)$.

Using this fact, we can introduce graded exterior forms on the simple graded manifold $\left(X, \mathfrak{A}_{Q}\right)$ as sections of the exterior bundle $\wedge \mathcal{V}_{Q}^{*}$, where $\mathcal{V}_{Q}^{*} \rightarrow X$ is the $\wedge Q^{*}$-dual of $\mathcal{V}_{Q}$. They are characterized both by the Grassmann parity and the familiar form degree. Relative to the dual local bases $\left\{d x^{\lambda}\right\}$ for $T^{*} X$ and $\left\{d c^{b}\right\}$ for $Q^{*}$, graded one-forms read

$$
\phi=\phi_{\lambda} d x^{\lambda}+\phi_{a} d c^{a}, \quad \phi_{a}^{\prime}=\rho_{a}^{-1 b} \phi_{b}, \quad \phi_{\lambda}^{\prime}=\phi_{\lambda}+\rho_{a}^{-1 b} \partial_{\lambda}\left(\rho_{j}^{a}\right) \phi_{b} c^{j},
$$

where $d x^{\lambda}$ are even and $d c^{b}$ are odd. The duality morphism is given by the interior product

$$
u\rfloor \phi=u^{\lambda} \phi_{\lambda}+(-1)^{\left[\phi_{a}\right]} u^{a} \phi_{a} .
$$

Graded exterior forms constitute the bigraded differential algebra (henceforth BGDA) $\mathcal{C}_{Q}^{*}$ with respect to the bigraded exterior product

$$
\phi \wedge \phi^{\prime}=(-1)^{|\phi|\left|\phi^{\prime}\right|+[\phi]\left[\phi^{\prime}\right]} \phi^{\prime} \wedge \phi, \quad \phi, \phi^{\prime} \in \mathcal{C}_{Q}^{*},
$$

and the exterior differential

$$
d\left(\phi \wedge \phi^{\prime}\right)=d \phi \wedge \phi^{\prime}+(-1)^{|\phi|} \phi \wedge d \phi^{\prime}, \quad \phi, \phi^{\prime} \in \mathcal{C}_{Q}^{*}
$$


Since the jet bundle $J^{r} Q \rightarrow X$ of a vector bundle $Q \rightarrow X$ is a vector bundle, let us consider the simple graded manifold $\left(X, \mathfrak{A}_{J^{r} Q}\right)$ constructed from $J^{r} Q \rightarrow X$. Its local basis is $\left\{x^{\lambda}, c_{\Lambda}^{a}\right\}, 0 \leq|\Lambda| \leq r$, together with the transition functions

$$
c_{\lambda+\Lambda}^{\prime a}=d_{\lambda}\left(\rho_{j}^{a} c_{\Lambda}^{j}\right), \quad d_{\lambda}=\partial_{\lambda}+\sum_{|\Lambda|<r} c_{\lambda+\Lambda}^{a} \partial_{a}^{\Lambda},
$$

where the graded derivations $\partial_{a}^{\Lambda}$ are the duals of $c_{\Lambda}^{a}$. Let $\mathcal{C}_{J^{r} Q}^{*}$ be the BGDA of graded exterior forms on the graded manifold $\left(X, \mathfrak{A}_{J^{r} Q}\right)$. A linear bundle morphism $\pi_{r-1}^{r}: J^{r} Q \rightarrow$ $J^{r-1} Q$ yields the corresponding monomorphism of BGDAs $\mathcal{C}_{J^{r-1} Q}^{*} \rightarrow \mathcal{C}_{J^{r} Q}^{*}$. Hence, there is the direct system of BGDAs

$$
\mathcal{C}_{Q}^{*} \stackrel{\pi_{0}^{1 *}}{\longrightarrow} \mathcal{C}_{J^{1} Q}^{*} \cdots \stackrel{\pi_{r-1}^{r}}{\longrightarrow} \mathcal{C}_{J^{r} Q}^{*} \longrightarrow \cdots
$$

Its direct limit $\mathcal{C}_{\infty}^{*} Q$ consists of graded exterior forms on graded manifolds $\left(X, \mathfrak{A}_{J^{r} Q}\right), r \in \mathbb{N}$, modulo the pull-back identification, and it inherits the BGDA operations intertwined by the monomorphisms $\pi_{r-1}^{r}{ }^{*}$. It is locally a free $C^{\infty}(X)$-algebra locally generated by the elements $\left(1, c_{\Lambda}^{a}, d x^{\lambda}, \theta_{\Lambda}^{a}=d c_{\Lambda}^{a}-c_{\lambda+\Lambda}^{a} d x^{\lambda}\right), 0 \leq|\Lambda|$, where $c_{\Lambda}^{a}$ and $\theta_{\Lambda}^{a}$ are odd.

In order to regard even and odd dynamic variables on the same footing, let $Y \rightarrow X$ be hereafter an affine bundle, and let $\mathcal{P}_{\infty}^{*} Y \subset \mathcal{O}_{\infty}^{*} Y$ be the $C^{\infty}(X)$-subalgebra of exterior forms whose coefficients are polynomial in the fiber coordinates on jet bundles $J^{r} Y \rightarrow X$. This notion is intrinsic since any element of $\mathcal{O}_{\infty}^{*} Y$ is an exterior form on some finite order jet manifold and all jet bundles $J^{r} Y \rightarrow X$ are affine. One can think of the GDA $\mathcal{P}_{\infty}^{*} Y$ as being the BGDA whose elements are even. Let us consider the product

$$
\mathcal{S}_{\infty}^{*}[Q ; Y]=\mathcal{C}_{\infty}^{*} Q \wedge \mathcal{P}_{\infty}^{*} Y
$$

of bigraded algebras $\mathcal{C}_{\infty}^{*} Q$ and $\mathcal{P}_{\infty}^{*} Y$ over their common graded subalgebra $\mathcal{O}^{*} X$ of exterior forms on $X .{ }^{4}$ It consists of the elements

$$
\sum_{i} \psi_{i} \otimes \phi_{i}, \quad \sum_{i} \phi_{i} \otimes \psi_{i}, \quad \psi \in \mathcal{C}_{\infty}^{*} Q, \quad \phi \in \mathcal{P}_{\infty}^{*} Y
$$

modulo the commutation relations

$$
\begin{aligned}
& \psi \otimes \phi=(-1)^{|\psi||\phi|} \phi \otimes \psi, \quad \psi \in \mathcal{C}_{\infty}^{*} Q, \quad \phi \in \mathcal{P}_{\infty}^{*} Y, \\
& (\psi \wedge \sigma) \otimes \phi=\psi \otimes(\sigma \wedge \phi), \quad \sigma \in \mathcal{O}^{*} X \text {. }
\end{aligned}
$$

These elements are endowed with the total form degree $|\psi \otimes \phi|=|\psi|+|\phi|$ and the total Grassmann parity $[\psi \otimes \phi]=[\psi]$. Their multiplication

$$
(\psi \otimes \phi) \wedge\left(\psi^{\prime} \otimes \phi^{\prime}\right):=(-1)^{\left|\psi^{\prime}\right||\phi|}\left(\psi \wedge \psi^{\prime}\right) \otimes\left(\phi \wedge \phi^{\prime}\right) .
$$


obeys the relation

$$
\varphi \wedge \varphi^{\prime}=(-1)^{|\varphi|\left|\varphi^{\prime}\right|+[\varphi]\left[\varphi^{\prime}\right]} \varphi^{\prime} \wedge \varphi, \quad \varphi, \varphi^{\prime} \in \mathcal{S}_{\infty}^{*}[Q ; Y]
$$

and makes $\mathcal{S}_{\infty}^{*}[Q ; Y](53)$ into a bigraded $C^{\infty}(X)$-algebra. For instance, elements of the ring $\mathcal{S}_{\infty}^{0}[Q ; Y]$ are polynomials of odd $c_{\Lambda}^{a}$ and even $y_{\Lambda}^{i}$ with coefficients in $C^{\infty}(X)$.

The algebra $\mathcal{S}_{\infty}^{*}[Q ; Y]$ is provided with the exterior differential

$$
d(\psi \otimes \phi):=\left(d_{\mathcal{C}} \psi\right) \otimes \phi+(-1)^{|\psi|} \psi \otimes\left(d_{\mathcal{P}} \phi\right), \quad \psi \in \mathcal{C}_{\infty}^{*}, \quad \phi \in \mathcal{P}_{\infty}^{*},
$$

where $d_{\mathcal{C}}$ and $d_{\mathcal{P}}$ are exterior differentials on the differential algebras $\mathcal{C}_{\infty}^{*} Q$ and $\mathcal{P}_{\infty}^{*} Y$, respectively. It obeys the relations

$$
d\left(\varphi \wedge \varphi^{\prime}\right)=d \varphi \wedge \varphi^{\prime}+(-1)^{|\varphi|} \varphi \wedge d \varphi^{\prime}, \quad \varphi, \varphi^{\prime} \in \mathcal{S}_{\infty}^{*}[Q ; Y]
$$

and makes $\mathcal{S}_{\infty}^{*}[Q ; Y]$ into a BGDA, which is locally generated by the elements

$$
\left(1, c_{\Lambda}^{a}, y_{\Lambda}^{i}, d x^{\lambda}, \theta_{\Lambda}^{a}=d c_{\Lambda}^{a}-c_{\lambda+\Lambda}^{a} d x^{\lambda}, \theta_{\Lambda}^{i}=d y_{\Lambda}^{i}-y_{\lambda+\Lambda}^{i} d x^{\lambda}\right), \quad 0 \leq|\Lambda|,
$$

where $c_{\Lambda}^{a}, \theta_{\Lambda}^{a}$ are odd and $y_{\Lambda}^{i}, d x^{\lambda}, \theta_{\Lambda}^{i}$ are even. The cohomology of its de Rham complex

$$
0 \rightarrow \mathbb{R} \longrightarrow \mathcal{S}_{\infty}^{0}[Q ; Y] \stackrel{d}{\longrightarrow} \mathcal{S}_{\infty}^{1}[Q ; Y] \cdots \stackrel{d}{\longrightarrow} \mathcal{S}_{\infty}^{k}[Q ; Y] \longrightarrow \cdots
$$

equals the de Rham cohomology $H^{*}(X)$ of $X .{ }^{4}$ We agree to call elements of $\mathcal{S}_{\infty}^{*}[Q ; Y]$ the graded exterior forms on $X$.

Hereafter, let the collective symbols $s_{\Lambda}^{A}$ and $\theta_{\Lambda}^{A}$ stand both for even and odd generating elements $c_{\Lambda}^{a}, y_{\Lambda}^{i}, \theta_{\Lambda}^{a}, \theta_{\Lambda}^{i}$ of the $C^{\infty}(X)$-algebra $\mathcal{S}_{\infty}^{*}[Q ; Y]$ which, thus, is locally generated by $\left(1, s_{\Lambda}^{A}, d x^{\lambda}, \theta_{\Lambda}^{A}\right),|\Lambda| \geq 0$. Since $s_{\Lambda}^{A}=d_{\Lambda} s^{A}$ and $\theta_{\Lambda}^{A}=d s_{\Lambda}^{A}+s_{\lambda+\Lambda}^{A} d x^{\lambda}$, the BGDA $\mathcal{S}_{\infty}^{*}[Q ; Y]$ is completely specified by the elements $s^{A}$ together with their transition functions. Therefore, we agree to call $\left\{s^{A}\right\}$ the local basis for $\mathcal{S}_{\infty}^{*}[Q ; Y]$.

Similarly to $\mathcal{O}_{\infty}^{*} Y$, the BGDA $\mathcal{S}_{\infty}^{*}[Q ; Y]$ is decomposed into $\mathcal{S}_{\infty}^{0}[Q ; Y]$-modules $\mathcal{S}_{\infty}^{k, r}[Q ; Y]$ of $k$-contact and $r$-horizontal graded forms together with the corresponding projections $h_{k}$ and $h^{r}$. Accordingly, the exterior differential $d(56)$ on $\mathcal{S}_{\infty}^{*}[Q ; Y]$ is split into the sum $d=d_{H}+d_{V}$ of the total and vertical differentials

$$
d_{H}(\phi)=d x^{\lambda} \wedge d_{\lambda}(\phi), \quad d_{V}(\phi)=\theta_{\Lambda}^{A} \wedge \partial_{A}^{\Lambda} \phi, \quad \phi \in \mathcal{S}_{\infty}^{*}[Q ; Y]
$$

The projection endomorphism $\varrho$ of $\mathcal{S}_{\infty}^{*}[Q ; Y]$ is given by the expression

$$
\left.\varrho=\sum_{k>0} \frac{1}{k} \bar{\varrho} \circ h_{k} \circ h^{n}, \quad \bar{\varrho}(\phi)=\sum_{0 \leq|\Lambda|}(-1)^{|\Lambda|} \theta^{A} \wedge\left[d_{\Lambda}\left(\partial_{A}^{\Lambda}\right\rfloor \phi\right)\right], \quad \phi \in \mathcal{S}_{\infty}^{>0, n}[Q ; Y],
$$


similar to (9). The graded variational operator $\delta=\varrho \circ d$ is introduced. Then the BGDA $\mathcal{S}_{\infty}^{*}[Q ; Y]$ is split into the $\mathbb{Z}_{2}$-graded variational bicomplex analogous to the above mentioned variational bicomplex of $\mathcal{O}_{\infty}^{*} Y$.

We restrict our consideration to the short variational subcomplex

$$
0 \longrightarrow \mathbb{R} \longrightarrow \mathcal{S}_{\infty}^{0}[Q ; Y] \stackrel{d_{H}}{\longrightarrow} \mathcal{S}_{\infty}^{0,1}[Q ; Y] \cdots \stackrel{d_{H}}{\longrightarrow} \mathcal{S}_{\infty}^{0, n}[Q ; Y] \stackrel{\delta}{\longrightarrow} \mathbf{E}_{1}
$$

and the subcomplex of one-contact graded forms

$$
0 \rightarrow \mathcal{S}_{\infty}^{1,0}[Q ; Y] \stackrel{d_{H}}{\longrightarrow} \mathcal{S}_{\infty}^{1,1}[Q ; Y] \cdots \stackrel{d_{H}}{\longrightarrow} \mathcal{S}_{\infty}^{1, n}[Q ; Y] \stackrel{\varrho}{\longrightarrow} \mathbf{E}_{1} \rightarrow 0
$$

of the BGDA $\mathcal{S}_{\infty}^{*}[Q ; Y]$. They possess the following cohomology. ${ }^{4}$

Theorem 10: The cohomology of the complex (58) equals the de Rham cohomology $H^{*}(X)$ of $X$.

Theorem 11: The complex (59) is exact.

One can think of the elements

$$
L=\mathcal{L} \omega \in \mathcal{S}_{\infty}^{0, n}[Q ; Y], \quad \delta L=\theta^{A} \wedge \mathcal{E}_{A} \omega=\sum_{0 \leq|\Lambda|}(-1)^{|\Lambda|} \theta^{A} \wedge d_{\Lambda}\left(\partial_{A}^{\Lambda} L\right) \omega \in \mathbf{E}_{1}
$$

of the complexes (58) - (59) as being a graded Lagrangian and its Euler-Lagrange operator, respectively. The components $\mathcal{E}_{A}$ of $\delta L$ are graded variational derivatives.

By virtue of Theorem 10, every $\delta$-closed graded Lagrangian $L(60)$ is the sum

$$
\phi=\psi+d_{H} \xi, \quad \xi \in \mathcal{S}_{\infty}^{0, n-1}[Q ; Y]
$$

where $\psi$ is a non-exact $n$-form on $X$.

The global exactness of the complex $(59)$ at the term $\mathcal{S}_{\infty}^{1, n}[Q ; Y]$ results in the following. ${ }^{4}$ Proposition 12: Given a graded Lagrangian $L=\mathcal{L} \omega$, there is the decomposition

$$
\begin{aligned}
& d L=\delta L-d_{H} \Xi, \quad \Xi \in \mathcal{S}_{\infty}^{1, n-1}[Q ; Y], \\
& \Xi=\sum_{s=0} \theta_{\nu_{s} \ldots \nu_{1}}^{A} \wedge F_{A}^{\lambda \nu_{s} \ldots \nu_{1}} \omega_{\lambda}, \quad F_{A}^{\nu_{k} \ldots \nu_{1}}=\partial_{A}^{\nu_{k} \ldots \nu_{1}} \mathcal{L}-d_{\lambda} F_{A}^{\lambda \nu_{k} \ldots \nu_{1}}+h_{A}^{\nu_{k} \ldots \nu_{1}},
\end{aligned}
$$

where local graded functions $h$ obey the relations $h_{a}^{\nu}=0, h_{a}^{\left(\nu_{k} \nu_{k-1}\right) \ldots \nu_{1}}=0$.

Proposition 12 states the existence of a global finite order Lepagean equivalent $\Xi_{L}=$ $\Xi+L$ of any graded Lagrangian $L$. Locally, one can always choose $\Xi$ (63) where all functions $h$ vanish. 


\section{BRST SYMMETRIES}

A graded derivation $\vartheta \in \mathfrak{d} \mathcal{S}_{\infty}^{0}[Q ; Y]$ of the $\mathbb{R}$-ring $\mathcal{S}_{\infty}^{0}[Q ; Y]$ is said to be contact if the Lie derivative $\mathbf{L}_{\vartheta}$ preserves the ideal of contact graded forms of the BGDA $\mathcal{S}_{\infty}^{*}[Q ; Y]$. With respect to the local basis $\left\{s^{A}\right\}$ for the BGDA $\mathcal{S}_{\infty}^{*}[Q ; Y]$, any contact graded derivation takes the form

$$
\vartheta=\vartheta_{H}+\vartheta_{V}=\vartheta^{\lambda} d_{\lambda}+\left(\vartheta^{A} \partial_{A}+\sum_{|\Lambda|>0} d_{\Lambda} \vartheta^{A} \partial_{A}^{\Lambda}\right),
$$

where tuple of graded derivations $\left\{\partial_{\lambda}, \partial_{A}^{\Lambda}\right\}$ is defined as the dual of the tuple $\left\{d x^{\lambda}, d s_{\Lambda}^{A}\right\}$ of generating elements of the $\mathcal{S}_{\infty}^{0}[Q ; Y]$-algebra $\mathcal{S}_{\infty}^{*}[Q ; Y]$, and $\vartheta^{\lambda}, \vartheta^{A}$ are local graded functions. ${ }^{4}$ The interior product $\left.\vartheta\right\rfloor \phi$ and the Lie derivative $\mathbf{L}_{\vartheta} \phi, \phi \in \mathcal{S}_{\infty}^{*}[Q ; Y]$, are defined by the same formulae

$$
\begin{aligned}
& \vartheta\rfloor \phi=\vartheta^{\lambda} \phi_{\lambda}+(-1)^{\left[\phi_{A}\right]} \vartheta^{A} \phi_{A}, \quad \phi \in \mathcal{S}_{\infty}^{1}[Q ; Y], \\
& \left.\vartheta\rfloor(\phi \wedge \sigma)=(\vartheta\rfloor \phi) \wedge \sigma+(-1)^{|\phi|+[\phi][\vartheta]} \phi \wedge(\vartheta\rfloor \sigma\right), \quad \phi, \sigma \in \mathcal{S}_{\infty}^{*}[Q ; Y], \\
& \left.\left.\mathbf{L}_{\vartheta} \phi=\vartheta\right\rfloor d \phi+d(\vartheta\rfloor \phi\right), \quad \mathbf{L}_{\vartheta}(\phi \wedge \sigma)=\mathbf{L}_{\vartheta}(\phi) \wedge \sigma+(-1)^{[\vartheta][\phi]} \phi \wedge \mathbf{L}_{\vartheta}(\sigma),
\end{aligned}
$$

as those on a graded manifold. One can justify that any vertical contact graded derivation $\vartheta(64)$ satisfies the relations

$$
\left.\vartheta\rfloor d_{H} \phi=-d_{H}(\vartheta\rfloor \phi\right), \quad \mathbf{L}_{\vartheta}\left(d_{H} \phi\right)=d_{H}\left(\mathbf{L}_{\vartheta} \phi\right), \quad \phi \in \mathcal{S}_{\infty}^{*}[Q ; Y]
$$

Proposition 13: It follows from the splitting (12) that the Lie derivative $\mathbf{L}_{\vartheta} L$ of a Lagrangian $L$ along a contact graded derivation $\vartheta(64)$ fulfills the first variational formula

$$
\left.\left.\left.\mathbf{L}_{\vartheta} L=\vartheta_{V}\right\rfloor \delta L+d_{H}\left(h_{0}(\vartheta\rfloor \Xi_{L}\right)\right)+d_{V}\left(\vartheta_{H}\right\rfloor \omega\right) \mathcal{L}
$$

where $\Xi_{L}=\Xi+L$ is a Lepagean equivalent of $L$ given by the coordinate expression (63). ${ }^{4}$

A contact graded derivation $\vartheta$ is said to be variational if the Lie derivative $(66)$ is $d_{H^{-}}$ exact. A glance at the expression (66) shows that: (i) a contact graded derivation $\vartheta$ is variational only if it is projected onto $X$, (ii) $\vartheta$ is variational iff its vertical part $\vartheta_{V}$ is variational.

Therefore, we restrict our consideration to vertical contact graded derivations

$$
\vartheta=\sum_{0 \leq|\Lambda|} d_{\Lambda} v^{A} \partial_{A}^{\Lambda},
$$


where the tuple of graded derivations $\left\{\partial_{A}^{\Lambda}\right\}$ is defined as the dual of the tuple $\left\{\theta_{\Lambda}^{A}\right\}$ of contact graded forms. Such a derivation is completely determined by its first summand

$$
v=v^{A}\left(x^{\lambda}, s_{\Lambda}^{A}\right) \partial_{A}, \quad 0 \leq|\Lambda| \leq k
$$

which is also a graded derivation of $\mathcal{S}_{\infty}^{0}[Q ; Y]$. It is called the generalized graded vector field. A glance at the first variational formula (66) shows that $\vartheta(67)$ is variational iff $v\rfloor \delta L$ is $d_{H-\text { exact. }}$

A vertical contact graded derivation $\vartheta(67)$ is said to be nilpotent if

$$
\mathbf{L}_{v}\left(\mathbf{L}_{v} \phi\right)=\sum_{|\Sigma| \geq 0,|\Lambda| \geq 0}\left(v_{\Sigma}^{B} \partial_{B}^{\Sigma}\left(v_{\Lambda}^{A}\right) \partial_{A}^{\Lambda}+(-1)^{\left[s^{B}\right]\left[v^{A}\right]} v_{\Sigma}^{B} v_{\Lambda}^{A} \partial_{B}^{\Sigma} \partial_{A}^{\Lambda}\right) \phi=0
$$

for any horizontal graded form $\phi \in \mathcal{S}_{\infty}^{0, *}[Q ; Y]$. One can show ${ }^{4}$ that $\vartheta$ is nilpotent only if it is odd and iff the equality

$$
\mathbf{L}_{\vartheta}\left(v^{A}\right)=\sum_{|\Sigma| \geq 0} v_{\Sigma}^{B} \partial_{B}^{\Sigma}\left(v^{A}\right)=0
$$

holds for all $v^{A}$.

Example 5: Let $Y \rightarrow X$ be an affine bundle, $L$ (4) a Lagrangian of a gauge theory on $Y$ and $v(24)$ its gauge symmetry where $E=Y \underset{X}{\times} V$ is the pull-back onto $Y$ of a vector bundle $V \rightarrow X$ coordinated by $\left(x^{\lambda}, \xi^{r}\right)$. Let us consider the BGDA $\mathcal{S}_{\infty}^{*}[V ; Y]=\mathcal{C}_{\infty}^{*} V \wedge \mathcal{P}_{\infty}^{*} Y$ possessing a local basis $\left\{c^{r}, y^{i}\right\}$. Let $L \in \mathcal{O}_{\infty}^{0, n} Y$ be a polynomial in $y_{\Lambda}^{i}, 0 \leq|L|$. Then it is a graded Lagrangian $L \in \mathcal{P}_{\infty}^{0, n} Y \subset \mathcal{S}_{\infty}^{0, n}[V ; Y]$ in $\mathcal{S}_{\infty}^{*}[V ; Y]$. Since $E \rightarrow Y$ is the pull-back bundle, a gauge symmetry $v(24)$ gives rise to the generalized vector field $v_{E}=v$ on $E$, and the latter defines the generalized graded vector field $v(68)$ by the formula

$$
v=\sum_{0 \leq|\Xi| \leq m} v_{r}^{i, \Xi}\left(x^{\lambda}, y_{\Sigma}^{i}\right) c_{\Xi}^{r} \partial_{i}
$$

It is easily justified that the vertical contact graded derivation $\vartheta(67)$ generated by $v(71)$ is variational for the graded Lagrangian $L$. It is odd, but need not be nilpotent. However, one can try to find a nilpotent contact graded derivation generated by some generalized graded vector field

$$
v=\sum_{0 \leq|\Lambda| \leq m} v_{r}^{i, \Lambda} c_{\Lambda}^{r} \partial_{i}+u^{r} \partial_{r}
$$

which coincides with $\vartheta$ on $\mathcal{P}_{\infty}^{*} Y$. In this case, the nilpotency conditions (70) read

$$
\begin{aligned}
& \sum_{\Sigma} d_{\Sigma}\left(\sum_{\Xi} v_{r}^{i, \Xi} c_{\Xi}^{r}\right) \sum_{\Lambda} \partial_{i}^{\Sigma}\left(v_{s}^{j, \Lambda}\right) c_{\Lambda}^{s}+\sum_{\Lambda} d_{\Lambda}\left(u^{r}\right) v_{r}^{j, \Lambda}=0, \\
& \sum_{\Lambda}\left(\sum_{\Xi} d_{\Lambda}\left(v_{r}^{i, \Xi} c_{\Xi}^{r}\right) \partial_{i}^{\Lambda}+d_{\Lambda}\left(u^{r}\right) \partial_{r}^{\Lambda}\right) u^{q}=0
\end{aligned}
$$


for all indices $j$ and $q$. They are equations for graded functions $u^{r} \in \mathcal{S}_{\infty}^{0}[V ; Y]$. Since these functions are polynomials

$$
u^{r}=u_{(0)}^{r}+\sum_{\Gamma} u_{(1) p}^{r, \Gamma} c_{\Gamma}^{p}+\sum_{\Gamma_{1}, \Gamma_{2}} u_{(2) p_{1} p_{2}}^{r, \Gamma_{1} \Gamma_{2}} c_{\Gamma_{1}}^{p_{1}} c_{\Gamma_{2}}^{p_{2}}+\cdots
$$

in $c_{\Lambda}^{s}$, the equations $(73)-(74)$ take the form

$$
\begin{aligned}
& \sum_{\Sigma} d_{\Sigma}\left(\sum_{\Xi} v_{r}^{i, \Xi} c_{\Xi}^{r}\right) \sum_{\Lambda} \partial_{i}^{\Sigma}\left(v_{s}^{j, \Lambda}\right) c_{\Lambda}^{s}+\sum_{\Lambda} d_{\Lambda}\left(u_{(2)}^{r}\right) v_{r}^{j, \Lambda}=0, \\
& \sum_{\Lambda} d_{\Lambda}\left(u_{(k \neq 2)}^{r}\right) v_{r}^{j, \Lambda}=0, \\
& \sum_{\Lambda} \sum_{\Xi} d_{\Lambda}\left(v_{r}^{i, \Xi} c_{\Xi}^{r}\right) \partial_{i}^{\Lambda} u_{(k-1)}^{q}+\sum_{m+n-1=k} d_{\Lambda}\left(u_{(m)}^{r}\right) \partial_{r}^{\Lambda} u_{(n)}^{q}=0 .
\end{aligned}
$$

One can think of the equalities (76) and (78) (and, consequently, the nilpotency conditions (73) - (74)) as being the generalized commutation relations and generalized Jacobi identities of gauge transformations, respectively. ${ }^{17}$ For instance, let us consider a gauge system on a principal bundle and the generalized vector field $v$ (44) in Example 4. Following the procedure above, we replace parameters $\xi^{r}$ and $\tau^{\lambda}$ with the odd ghosts $C^{r}$ and $C^{\lambda}$, respectively, and obtain the generalized graded vector field

$$
v=\left(c_{p q}^{r} a_{\lambda}^{p} C^{q}+C_{\lambda}^{r}-a_{\mu}^{r} C_{\lambda}^{\mu}-C^{\mu} a_{\mu \lambda}^{r}\right) \partial_{r}^{\lambda}+\left(-\frac{1}{2} c_{p q}^{r} C^{p} C^{q}-C^{\mu} C_{\mu}^{r}\right) \partial_{r}+C_{\mu}^{\lambda} C^{\mu} \partial_{\lambda}
$$

such that the vertical contact graded derivation (67) generated by $v(79)$ is nilpotent. In the case of the vertical gauge symmetry (46), we obtain the familiar BRST transformation

$$
v=\left(c_{p q}^{r} a_{\lambda}^{p} C^{q}+C_{\lambda}^{r}\right) \partial_{r}^{\lambda}-\frac{1}{2} c_{p q}^{r} C^{p} C^{q} \partial_{r}
$$

of Yang-Mills theory.

Generalizing Example 5, we describe BRST symmetries in a general setting as follows.

Let $\mathcal{S}_{\infty}^{*}[Q ; Y]$ be the BGDA (53) and $L \in \mathcal{S}_{\infty}^{0, n}[Q ; Y]$ a graded Lagrangian. We agree to call generating elements $s^{A}$ of $\mathcal{S}_{\infty}^{*}[Q ; Y]$ the fields. Let $V \rightarrow X$ be a vector bundle coordinated by $\left(x^{\lambda}, \xi^{r}\right)$. By analogy with $\mathcal{S}_{\infty}^{*}[Q ; Y]$, we consider the BGDA

$$
\mathcal{S}_{\infty}^{*}[Q V ; Y]=\mathcal{C}_{\infty}^{*}[Q \underset{X}{\times} V] \wedge \mathcal{P}_{\infty}^{*} Y
$$

whose local basis is $\left\{s^{A}, c^{r}\right\}$. Obviously, $L$ is also a graded Lagrangian in $\mathcal{S}_{\infty}^{*}[Q V ; Y]$. Let

$$
\vartheta=\sum_{0 \leq|\Lambda|}\left(d_{\Lambda} v^{A} \partial_{A}^{\Lambda}+d_{\Lambda} v^{r} \partial_{r}^{\Lambda}\right)
$$


be a graded contact derivation of the $\mathbb{R}$-ring $\mathcal{S}_{\infty}^{0}[Q V ; Y]$ generated by an odd generalized graded vector field

$$
v=v^{A} \partial_{A}+v^{r} \partial_{r}
$$

whose restriction to $\mathcal{S}_{\infty}^{*}[Q ; Y]$ is linear in $c_{\Lambda}^{r}$, i.e.,

$$
v=\sum_{0 \leq|\Xi| \leq m} c_{\Xi}^{r} v_{r}^{A, \Xi}\left(x^{\lambda}, s_{\Sigma}^{B}\right) \partial_{A}+v^{r} \partial_{r} .
$$

If $\vartheta(82)$ is variational for $L$ and nilpotent, we say that $v(84)$ is a BRST symmetry of $L$. Following the terminology of BRST theory, we agree to call generating elements $c^{r}$ of $\mathcal{S}^{*}[Q V ; Y]$ the ghosts.

\section{NOETHER'S SECOND THEOREM II}

In order to introduce Noether identities in the case of BRST symmetries, let us extend the BGDA $\mathcal{S}^{*}[Q V ; Y](81)$ to the BGDA

$$
\mathcal{S}_{\infty}^{*}\left[Q \bar{Y}^{*} V ; Y \bar{Q}^{*}\right]=\mathcal{C}_{\infty}^{*}\left[Q \underset{X}{\times} \bar{Y}_{X}^{*} \underset{X}{\times} V\right] \wedge \mathcal{P}_{\infty}^{*}\left[Y \underset{X}{\times} \bar{Q}^{*}\right],
$$

where $\bar{Q}^{*}$ is the density-dual of $Q$ and $\bar{Y}^{*}$ is the density dual of the vector bundle $\tilde{Y} \rightarrow X$ which the affine bundle $Y$ is modelled on (e.g., $\tilde{Y}=Y$ if $Y$ is a vector bundle). The local basis for the BGDA $\mathcal{S}_{\infty}^{*}\left[Q \bar{Y}^{*} V ; Y \bar{Q}^{*}\right]$ is $\left\{s^{A}, c^{r}, \bar{s}_{A}\right\}$. Following the terminology of the fieldantified BRST theory, we call generating elements $\bar{s}_{A}$ of $\mathcal{S}_{\infty}^{*}\left[Q \bar{Y}^{*} V ; Y \bar{Q}^{*}\right]$ the antifields. Their Grassmann parity is $\left[\bar{s}_{A}\right]=\left(\left[s^{A}\right]+1\right) \bmod 2$.

The BGDA $\mathcal{S}_{\infty}^{*}\left[Q \bar{Y}^{*} V ; Y \bar{Q}^{*}\right](85)$ is provided with the Koszul-Tate differential defined as the nilpotent contact graded derivation

$$
\bar{\delta}=\sum_{0 \leq|\Lambda|} \overleftarrow{\partial}^{\Lambda A} d_{\Lambda} \mathcal{E}_{A}
$$

where $\mathcal{E}_{A}$ are the graded variational derivatives (60) and the tuple of graded right derivations $\overleftarrow{\partial}^{\Lambda A}$ is the dual of the tuple of contact graded forms $\left\{\theta_{\Lambda A}\right\}$, i.e.,

$$
\theta_{\Lambda A} \overleftarrow{L}^{\Sigma} \Sigma B=\delta_{\Lambda}^{\Sigma} \delta_{B}^{A}
$$

where multi-indices $\Sigma$ and $\Lambda$ are regard modulo permutations. Because of the expression (60) for $\delta L$, it is convenient to describe the Koszul-Tate differential as a graded derivation acting on graded functions and forms $\phi$ on the right by the rule

$$
\bar{\delta}(\phi)=d \phi\left[\bar{\delta}+d(\phi \mid \bar{\delta}), \quad \bar{\delta}\left(\phi \wedge \phi^{\prime}\right)=(-1)^{\left[\phi^{\prime}\right]} \bar{\delta}(\phi) \wedge \phi^{\prime}+\phi \wedge \bar{\delta}\left(f^{\prime}\right) .\right.
$$


Definition 14: Given a graded Lagrangian $L \in \mathcal{S}_{\infty}^{*}[Q ; Y] \subset \mathcal{S}_{\infty}^{*}\left[Q \bar{Y}^{*} V ; Y \bar{Q}^{*}\right](60)$, we say that its Euler-Lagrange operator $\delta L(60)$ obeys a Noether identity if there exists a $\bar{\delta}$-closed even graded density

$$
\Delta=c^{r} \Delta_{r} \omega=\sum_{0 \leq|\Lambda| \leq m} c^{r} \Delta_{r}^{A, \Lambda}\left(x^{\lambda}, s_{\Sigma}^{B}\right) \bar{s}_{\Lambda A} \omega \in \mathcal{S}_{\infty}^{0, n}\left[Q \bar{Y}^{*} V ; Y \bar{Q}^{*}\right]
$$

which is linear both in ghosts $c^{r}$ and antifields $\bar{s}_{A}$ and their jets $\bar{s}_{\Lambda A}$. The above mentioned Noether identity reads

$$
\bar{\delta}(\Delta)=c^{r}\left[\sum_{0 \leq|\Lambda| \leq m} \Delta_{r}^{A, \Lambda} d_{\Lambda} \mathcal{E}_{A}\right] \omega=0
$$

Then Noether's second theorem for BRST symmetries is formulated as follows.

Theorem 15: If $v(84)$ is a BRST symmetry of a graded Lagrangian $L$, then

$$
\begin{aligned}
& \Delta=\eta(v)=\sum_{0 \leq|\Lambda| \leq m} c^{r} \eta(v)_{r}^{A, \Lambda} \bar{s}_{\Lambda A} \omega \\
& \eta(v)_{r}^{A, \Lambda}=\sum_{0 \leq|\Sigma| \leq m-|\Lambda|}(-1)^{|\Sigma+\Lambda|} C_{|\Sigma+\Lambda|}^{|\Sigma|} d_{\Sigma} v_{r}^{A, \Sigma+\Lambda}
\end{aligned}
$$

is a $\bar{\delta}$-closed graded density (87). Conversely, if a $\bar{\delta}$-closed graded density $\Delta(87)$ exists, the generalized graded vector field

$$
\begin{aligned}
& v=\eta(\Delta)=\sum_{0 \leq|\Xi| \leq m} c_{\Xi}^{r} \eta(\Delta)_{r}^{A, \Xi} \partial_{A}, \\
& \eta(\Delta)_{r}^{A, \Lambda}=\sum_{0 \leq|\Sigma| \leq m-|\Lambda|}(-1)^{|\Sigma+\Lambda|} C_{|\Sigma+\Lambda|}^{|\Sigma|} d_{\Sigma} \Delta_{r}^{A, \Sigma+\Lambda},
\end{aligned}
$$

generates a contact graded derivation (82) which is variational for the graded Lagrangian $L$, but it need not be nilpotent. The relations (2) hold.

Proof: The first summand of the generalized vector field (84) defines the graded function

$$
v=\sum_{0 \leq|\Xi| \leq m} c_{\Xi}^{r} v_{r}^{A, \Xi}\left(x^{\lambda}, s_{\Sigma}^{B}\right) s_{A} \in \mathcal{S}_{\infty}^{*}\left[Q Q^{*} V ; Y \tilde{Y}^{*}\right]
$$

and vice versa. Then the proof follows from Theorem 18 in Appendix B. By virtue of this theorem, the graded function (91) yields the graded density (89). Since the graded density $v\rfloor \delta L$ is $d_{H^{-}}$-exact, we obtain the equality

$$
\delta(v\rfloor \delta L)=\bar{\delta}(\eta(v))=0
$$


Conversely, the graded density (87) yields the graded function (91) where $v_{r}^{A, \Xi}=\eta(\Delta)_{r}^{A, \Xi}$. Since $\Delta(87)$ is $\bar{\delta}$-closed, we have

$$
\begin{aligned}
0= & \sum_{0 \leq|\Lambda| \leq m} c^{r} \Delta_{r}^{A, \Lambda} d_{\Lambda} \mathcal{E}_{A} \omega=\sum_{0 \leq|\Lambda| \leq m}(-1)^{|\Lambda|} d_{\Lambda}\left(c^{r} \Delta_{r}^{A, \Lambda}\right) \mathcal{E}_{A} \omega+d_{H} \sigma= \\
& \left.\sum_{0 \leq|\Xi| \leq m} c_{\Xi}^{r} v_{r}^{A, \Xi} \mathcal{E}_{A} \omega+d_{H} \sigma=v\right\rfloor \delta L+d_{H} \sigma,
\end{aligned}
$$

i.e., the graded contact derivation generated by $v(90)$ is variational for $L$. Due to the equality (32), the relations (2) hold.

Bearing in mind the field-antifield BRST theory and BV quantization, the Noether identity (88) can be rewritten as follows. Let us consider the BGDA $\mathcal{S}_{\infty}^{*}\left[Q \bar{Y}^{*} V ; Y \bar{Q}^{*} \bar{V}^{*}\right]$ possessing the local basis $\left\{s^{A}, c^{r}, \bar{s}_{A}, \bar{c}_{r}\right\}$, where even elements $\bar{c}_{r}$ are called antighosts of the ghosts $c^{r}$. Clearly, the graded density $\Delta(87)$ is an element of $\mathcal{S}_{\infty}^{*}\left[Q \bar{Y}^{*} V ; Y \bar{Q}^{*} \bar{V}^{*}\right]$. Then this BGDA is provided with the contact graded right derivation

$$
\bar{\delta}_{c}=\sum_{0 \leq|\Lambda|}\left(\overleftarrow{\partial}^{\Lambda A} d_{\Lambda} \mathcal{E}_{A}+\overleftarrow{\partial}^{\Lambda r} d_{\Lambda} \Delta_{r}\right)
$$

where the tuple of graded right derivations $\overleftarrow{\partial}^{\Lambda r}$ is the dual of $\theta_{\Lambda r}$. It is easily justified that the graded density $\Delta$ (87) obeys the Noether identity (88) iff the graded right derivation $\bar{\delta}_{c}(92)$ is nilpotent. It is the extension of the Koszul-Tate differential (86) to antighosts. For instance, the graded density $\Delta$ is always $\bar{\delta}_{c}$-exact.

\section{REDUCIBLE BRST SYMMETRIES}

The notion of a reducible Noether identity in Section IV is straightforwardly generalized to BRST symmetries, but we formulate it in terms of the Koszul-Tate differential. We say that the Noether identity (88) is $N$-stage reducible if the following conditions hold.

(a) There exists a set of vector bundles $V_{-1}=V, V_{0}, \ldots, V_{N}$ over $X$, and we consider the BGDA

$$
\begin{aligned}
& \overline{\mathcal{S}}_{\infty}^{*}\{N\}= \\
& \quad \mathcal{S}_{\infty}^{*}\left[Q \bar{Y}^{*} V V_{1} \ldots V_{2 k-1} \ldots \bar{V}_{0}^{*} \ldots \bar{V}_{2 k}^{*} \ldots ; Y \bar{Q}^{*} V_{0} \ldots V_{2 k} \ldots \bar{V}^{*} \bar{V}_{1}^{*} \ldots \bar{V}_{2 k-1}^{*} \ldots\right] .
\end{aligned}
$$

It possesses a local basis

$$
\left\{s^{A}, \bar{s}_{A}, c^{r}, c^{r_{0}}, \ldots, c^{r_{N}}, \bar{c}_{r}, \bar{c}_{r_{0}}, \ldots, \bar{c}_{r_{N}}\right\}, \quad\left[c^{r_{k}}\right]=k \bmod 2,
$$


where $c^{r_{k}}$ and $\bar{c}_{r_{k}}$ are called the $k$-stage ghosts and antighosts, respectively..

(b) The BGDA (93) contains the graded density $\Delta$ (87) and a set of even graded densities

$$
\Delta_{(k)}=c^{r_{k}} \Delta_{r_{k}} \omega=\sum_{\Lambda} c^{r_{k}} \Delta_{r_{k}}^{r_{k-1}, \Lambda}\left(x^{\lambda}, s_{\Sigma}^{B}\right) \bar{c}_{\Lambda r_{k-1}} \omega, \quad k=0, \ldots, N
$$

such that the contact graded derivation

$$
\bar{\delta}_{N}=\sum_{0 \leq|\Lambda|}\left(\overleftarrow{\partial}^{\Lambda A} d_{\Lambda} \mathcal{E}_{A}+\overleftarrow{\partial}^{\Lambda r} d_{\Lambda} \Delta_{r}+\overleftarrow{\partial}^{\Lambda r_{0}} d_{\Lambda} \Delta_{r_{0}}+\cdots+\overleftarrow{\partial}^{\Lambda r_{N}} d_{\Lambda} \Delta_{r_{N}}\right)
$$

is weakly nilpotent, i.e., $\bar{\delta}_{N}\left(\bar{\delta}_{N}(f)\right)$ is $\bar{\delta}$-exact for any graded function $f \in \overline{\mathcal{S}}_{\infty}^{0}\{N\}$. This nilpotency condition is equivalent to the requirement that all the compositions

$$
\sum_{\Xi} c^{r_{k}} \Delta_{r_{k}}^{r_{k-1}, \Xi} d_{\Xi}\left(\sum_{\Lambda} \Delta_{r_{k-1}}^{r_{k-2}, \Lambda} \bar{c}_{r_{k-2}}\right), \quad k=1, \ldots, N
$$

are $\bar{\delta}$-exact. The graded derivation $\bar{\delta}_{N}$ (96) is called the $N$-stage Koszul-Tate differential.

(c) No graded density $\Delta, \Delta_{(k)}, k=0, \ldots, N$, is $\bar{\delta}$-exact. Let $V_{0}^{\prime}, \ldots, V_{N^{\prime}}^{\prime}$ be another set of vector bundles such that $V_{k \leq N}^{\prime}$ contains $V_{k}$ as a direct summand. Then the corresponding BGDA $\overline{\mathcal{S}}_{\infty}^{*}\left\{N^{\prime}\right\}$ (93) contains the graded densities $\Delta(87), \Delta_{(k)}(95), k=0, \ldots, N$, and it is provided with the contact graded derivation $\bar{\delta}_{N}(96)$. If there exists another set $\Delta_{(k)}^{\prime}$, $k=0, \ldots, N^{\prime}$, of graded densities obeying the conditions in item (b), then any graded density $\Delta_{(k)}^{\prime}$ of this set is $\bar{\delta}_{N}$-exact.

Note, that following the arguments in Section III, one can say that the Noether identity (88) is complete if it obeys the condition (c).

If the Noether identity (88) is reducible, the associated BRST symmetry (90) is reducible as follows.

Let us consider the BGDA

$$
\mathcal{S}_{\infty}^{*}\{N\}=\mathcal{S}_{\infty}^{*}\left[Q Q^{*} V V^{*} V_{1} V_{1}^{*} \ldots V_{2 k-1} V_{2 k-1}^{*} \ldots ; Y \tilde{Y}^{*} V_{0} V_{0}^{*} \ldots V_{2 k} V_{2 k}^{*} \ldots\right]
$$

possessing the local basis $\left\{s^{A}, s_{A}, c^{r}, c^{r_{0}}, \ldots, c^{r_{N}}, c_{r}, c_{r_{0}}, \ldots, c_{r_{N}}\right\}$. By virtue of Theorem 18, each graded density $\Delta_{(k)}(95), k=0, \ldots, N$, defines the graded function

$$
v_{(k)}=\sum_{\Xi} c_{\Xi}^{r_{k}} \eta\left(\Delta_{(k)}\right)_{r_{k}}^{r_{k-1}, \Xi} c_{r_{k-1}} \in \mathcal{S}_{\infty}^{0}(\{N\}
$$

and, consequently, the generalized graded vector field

$$
v_{(k)}=\sum_{\Xi} c_{\Xi}^{r_{k}} \eta\left(\Delta_{(k)}\right)_{r_{k}}^{r_{k-1}, \Xi} \partial_{r_{k-1}} \in \mathcal{S}_{\infty}^{0}(\{N\}
$$


which yields a contact graded derivation of the BGDA $\overline{\mathcal{S}}_{\infty}^{*}\{N\}$. Similarly to the proof of Theorem 9, one can show that they possess the following properties.

(a') Contact graded derivations $\vartheta$ and $\vartheta_{(k)}$ generated by the generalized graded vector fields $v(90)$ and $v_{(k)}(99)$ are not weakly $\bar{\delta}$-exact, i.e., $\vartheta(f)$ and $\vartheta_{(k)}(f)$ are not $\bar{\delta}$-exact for some graded function $f \in \overline{\mathcal{S}}_{\infty}^{*}\{N\}$.

(b') Contact graded derivations generated by the generalized graded vector fields

$$
\begin{aligned}
& \sum_{\Lambda} d_{\Lambda}\left(\sum_{\Xi} c_{\Xi}^{r_{0}} \eta\left(\Delta_{(0)}\right)_{r_{0}}^{r, \Xi}\right) \eta(\Delta)_{r}^{A, \Lambda} \partial_{A}, \\
& \sum_{\Lambda} d_{\Lambda}\left(\sum_{\Xi} c_{\Xi}^{r_{k}} \eta\left(\Delta_{(k)}\right)_{r_{k}}^{r_{k-1}, \Xi}\right) \eta\left(\Delta_{(k-1)}\right)_{r_{k-1}}^{r_{k-2}, \Lambda} \partial_{r_{k-2}}, \quad k=1, \ldots, N,
\end{aligned}
$$

are weakly $\bar{\delta}$-exact. This condition can be reformulated as follows. Let us consider the BGDA

$$
\mathcal{S}_{\infty}^{*}\left[Q Q \bar{Y}^{*} V V_{1} \ldots V_{2 k-1} \ldots \bar{V}_{0}^{*} \ldots \bar{V}_{2 k}^{*} \ldots ; Y \tilde{Y} \bar{Q}^{*} V_{0} \ldots V_{2 k} \ldots \bar{V}^{*} \bar{V}_{1}^{*} \ldots \bar{V}_{2 k-1}^{*} \ldots\right]
$$

whose basis consists of elements (94) and the elements $s^{\prime A}$ associated to the additional bundles $Q$ and $\tilde{Y}$. Let us replace the generalized graded vector field $v(90)$ with

$$
v^{\prime}=\sum_{0 \leq|\Xi| \leq m} c_{\Xi}^{r} \eta(\Delta)_{r}^{A, \Xi}\left(x^{\lambda}, s_{\Sigma}^{B}\right) \partial_{A}^{\prime},
$$

where the graded derivations $\partial_{A}^{\prime}$ are dual of the basis elements $s^{\prime A}$. Then the contact graded derivation generated by the generalized graded vector field

$$
v_{N}=v^{\prime}+v_{(0)}+\cdots+v_{(N)}
$$

is weakly nilpotent.

(c') Let $v_{N^{\prime}}$ be the generalized graded vector field (103) defined by the graded densities $\Delta_{(k)}^{\prime}$ in item (c) above. Then there exists some generalized graded vector field $u$ such that $v_{N^{\prime}}-\left[u, v_{N}\right]$ is weakly $\bar{\delta}$-exact.

Conversely, one can show the following. Let $v(90)$ be a BRST symmetry of a graded Lagrangian $L$. Let us assume that there exists a set of generalized graded vector fields

$$
v_{(k)}=\sum_{\Xi} c_{\Xi}^{r_{k}} v_{r_{k}}^{r_{k-1}, \Xi} \partial_{r_{k-1}} \in \mathcal{S}_{\infty}^{0}\{N\}
$$

which obey the conditions in items $\left(a^{\prime}\right)-\left(c^{\prime}\right)$. Then the graded density (15) defines a complete reducible Noether identity where the graded densities

$$
\Delta_{(k)}=\sum_{\Lambda} c^{r_{k}} \eta\left(v_{(k)}\right)_{r_{k}}^{r_{k-1}, \Lambda}\left(x^{\lambda}, s_{\Sigma}^{B}\right) \bar{c}_{\Lambda r_{k-1}}
$$


obey the conditions in items $(\mathrm{a})-(\mathrm{c})$.

In contrast with the generalized graded vector field $v_{N}(103)$, the contact graded derivation generated by the generalized graded vector field $v+v_{(0)}+\cdots+v_{(N)}$ need not be weakly nilpotent. Its extension to the nilpotent one provides a BRST symmetry of the field-antifield BRST theory whose Lagrangian depends on ghosts and antifields.

\section{APPENDIX A}

A $k$-order differential operator on a fiber bundle $Y \rightarrow X$ with values in a fiber bundle $Z \rightarrow X$ is defined as a section $\Delta$ of the fiber bundle $J^{k} Y \underset{X}{\times} Z \rightarrow J^{k} Y$. It admits an $m$-order jet prolongation $\Delta^{(m)}$ as a section of the fiber bundle

$$
J^{m+k} Y \underset{X}{\times} J^{m} Z \rightarrow J^{m+k} Y
$$

By a differential operator throughout is meant its appropriate finite order jet prolongation. Given bundle coordinates $\left(x^{\lambda}, y^{i}\right)$ on $Y$ and $\left(x^{\lambda}, z^{A}\right)$ on $Z$, a differential operator $\Delta$ reads

$$
z^{A} \circ \Delta=\Delta^{A}\left(x^{\lambda}, y_{\Lambda}^{i}\right), \quad z_{\Sigma}^{A} \circ \Delta^{(m)}=d_{\Sigma} \Delta^{A}, \quad 0 \leq|\Lambda| \leq k, \quad 0 \leq|\Sigma| \leq m .
$$

If $Z$ is a composite fiber bundle $\pi \circ \pi_{Z Y}: Z \rightarrow Y \rightarrow X$ and the relation $\pi_{Z Y} \circ \Delta=\pi_{0}^{k}$ holds, a differential operator $\Delta$ is identified to a section of the fiber bundle $J^{k} Y \underset{Y}{\times} Z \rightarrow J^{k} Y$ or, equivalently, a bundle morphism $J^{k} Y \underset{Y}{\longrightarrow} Z$.

Let $E \rightarrow Y$ and $Q \rightarrow Y$ be vector bundles. A $k$-order $Q$-valued differential operator $v$ on $E \rightarrow X$ is called linear on $E \rightarrow Y$ (or, simply, linear) if $v: J^{k} E \rightarrow Q$ is a morphism of the vector bundle $J^{k} E \rightarrow J^{k} Y$ to the vector bundle $Q \rightarrow Y$ over $\pi_{0}^{k}: J^{k} Y \rightarrow Y$. Given bundle coordinates $\left(x^{\lambda}, y^{i}, \xi^{r}\right)$ on $E$ and $\left(x^{\lambda}, y^{i}, q^{a}\right)$ on $Q$, such an operator is represented by the function

$$
v=v^{a} q_{a}=\sum_{0 \leq|\Lambda| \leq m} v_{r}^{a, \Lambda}\left(x^{\lambda}, y_{\Sigma}^{i}\right) \xi_{\Lambda}^{r} q_{a} \in \mathcal{O}_{\infty}^{0}\left[\underset{Y}{E \times} Q^{*}\right], \quad 0 \leq|\Sigma| \leq m .
$$

Let us consider the density-dual $\bar{E}^{*}(1)$ of a vector bundle $E \rightarrow Y$ and that $\bar{Q}^{*}$ of $Q \rightarrow Y$ coordinated by $\left(x^{\lambda}, y^{i}, \bar{q}_{a}\right)$. Let $\Delta$ be a linear $\bar{E}^{*}$-valued differential operator on $\bar{Q}^{*}$. It is represented by the density

$$
\Delta=\Delta_{r} \xi^{r} \omega=\sum_{0 \leq|\Lambda| \leq m} \Delta_{r}^{a, \Lambda}\left(x^{\lambda}, y_{\Sigma}^{i}\right) \bar{q}_{\Lambda a} \xi^{r} \omega \in \mathcal{O}_{\infty}^{0, n}\left[\underset{Y}{E \underset{Y}{\times}} Q^{*}\right], \quad 0 \leq|\Sigma| \leq m
$$


Theorem 16: Any linear $Q$-valued differential operator $v(105)$ on $E$ yields the linear $\bar{E}^{*}$ valued differential operator

$$
\begin{aligned}
& \eta(v)=\sum_{0 \leq|\Lambda| \leq m} \eta(v)_{r}^{a, \Lambda} \bar{q}_{\Lambda a} \xi^{r} \omega \\
& \eta(v)_{r}^{a, \Lambda}=\sum_{0 \leq|\Sigma| \leq m-|\Lambda|}(-1)^{|\Sigma+\Lambda|} C_{|\Sigma+\Lambda|}^{|\Sigma|} d_{\Sigma}\left(v_{r}^{a, \Sigma+\Lambda}\right),
\end{aligned}
$$

on $\bar{Q}^{*}$. Conversely, any linear $\bar{E}^{*}$-valued differential operator $\Delta$ (106) on $\bar{Q}^{*}$ defines the linear $Q$-valued differential operator

$$
\begin{aligned}
& \eta(\Delta)=\sum_{0 \leq|\Lambda| \leq m} \eta(\Delta)_{r}^{a, \Lambda} \xi_{\Lambda}^{r} q_{a}, \\
& \eta(\Delta)_{r}^{a, \Lambda}=\sum_{0 \leq|\Sigma| \leq m-|\Lambda|}(-1)^{|\Sigma+\Lambda|} C_{|\Sigma+\Lambda|}^{|\Sigma|} d_{\Sigma}\left(\Delta_{r}^{a, \Sigma+\Lambda}\right),
\end{aligned}
$$

on $E$. The relations (2) hold.

Proof: The function $v(105)$ defines the density

$$
\bar{v}=\sum_{0 \leq|\Lambda| \leq m} v_{r}^{a, \Lambda} \xi_{\Lambda}^{r} \bar{q}_{a} \omega \in \mathcal{O}_{\infty}^{0, n}\left[E \underset{Y}{\times} Q^{*}\right]
$$

Its Euler-Lagrange operator

$$
\delta(\bar{v})=\mathcal{E}_{i} d y^{i} \wedge \omega+\mathcal{E}_{r} d \xi^{r} \wedge \omega+\mathcal{E}^{a} d \bar{q}_{a} \wedge \omega
$$

takes its values in the fiber bundle

$$
V^{*}\left(\underset{Y}{E \times} Q^{*}\right) \underset{E \times Q_{Y}^{*}}{\otimes} \wedge^{n} T^{*} X
$$

where $V^{*}\left(\underset{Y}{E} Q^{*}\right)$ is the vertical cotangent bundle of the fiber bundle $E \underset{Y}{E} Q^{*} \rightarrow X$. Let

$$
\alpha_{E}: V^{*}\left(\underset{Y}{\operatorname{Ex}} Q^{*}\right) \rightarrow V_{Y}^{*}\left(\underset{Y}{\operatorname{Ex}} Q^{*}\right) \rightarrow V_{Y}^{*} E
$$

be the canonical projection of $V^{*}\left(\underset{Y}{\underset{Y}{\times}} Q^{*}\right)$ onto the vertical cotangent bundle $V_{Y}^{*}\left(E \underset{Y}{E} Q^{*}\right)$ of the fiber bundle $E \underset{Y}{\times} Q^{*} \rightarrow Y$ and, afterwards, onto the vertical cotangent bundle $V_{Y}^{*} E$ of $E \rightarrow Y$. Then we obtain a differential operator $\left(\alpha_{E} \circ \delta\right)(\bar{v})$ on $\underset{Y}{\times} Q^{*}$ with values in the fiber bundle $V_{Y}^{*} E \underset{E}{\otimes} \wedge T^{*} X$. It reads

$$
\left(\alpha_{E} \circ \delta\right)(\bar{v})=\mathcal{E}_{r} \bar{d} \xi^{r} \otimes \omega=\sum_{0 \leq|\Lambda| \leq m}(-1)^{|\Lambda|} d_{\Lambda}\left(v_{r}^{a, \Lambda} \bar{q}_{a}\right) \bar{d} \xi^{r} \otimes \omega
$$


where $\left\{\bar{d} \xi^{r}\right\}$ is the fiber basis for $V_{Y}^{*} E \rightarrow E$. Due to the canonical isomorphism $V_{Y}^{*} E=$ $E^{*} \times \underset{Y}{E}$, this operator defines the density

$$
\sum_{0 \leq|\Lambda| \leq m}(-1)^{|\Lambda|} d_{\Lambda}\left(v_{r}^{a, \Lambda} \bar{q}_{a}\right) \xi^{r} \omega \in \mathcal{O}_{\infty}^{0, n}\left[\underset{Y}{\operatorname{x}} Q^{*}\right]
$$

and, by virtue of the formula (30), the desired differential operator (107). Conversely, the Euler-Lagrange operator of the density (106) takes its values in the fiber bundle (110) and reads

$$
\delta(\bar{v})=\mathcal{E}_{i} d y^{i} \wedge \omega+\mathcal{E}_{r} d \xi^{r} \wedge \omega+\mathcal{E}^{a} d \bar{q}_{a} \wedge \omega
$$

In order to repeat the above mentioned procedure, let us consider a volume form $J \omega$ on $X$ and substitute $d \bar{q}_{a} \wedge \omega=J d q_{a} \wedge \omega$ into the expression (112). Using the projection

$$
\alpha_{Q}: V^{*}\left(\underset{Y}{E \times} Q^{*}\right) \rightarrow V_{Y}^{*} Q^{*}
$$

similar to $\alpha_{E}(111)$ and the canonical isomorphism $V_{Y}^{*} Q^{*}=\underset{Y}{\underset{Y}{\times}} Q^{*}$, we come to the density

$$
\sum_{0 \leq|\Lambda| \leq m}(-1)^{|\Lambda|} d_{\Lambda}\left(\Delta_{r}^{a, \Lambda} \xi^{r}\right) q_{a} J \omega \in \mathcal{O}_{\infty}^{0, n}\left[\underset{Y}{E \times} Q^{*}\right]
$$

and, hence, the function

$$
\sum_{0 \leq|\Lambda| \leq m}(-1)^{|\Lambda|} d_{\Lambda}\left(\Delta_{r}^{a, \Lambda} \xi^{r}\right) q_{a} \in \mathcal{O}_{\infty}^{0}\left[E \underset{Y}{E \times} Q^{*}\right]
$$

defining the desired operator (108). The relations (2) result from the relation (32).

Relations (2) show that the intertwining operator $\eta(107)-(108)$ provides a bijection between the sets $\operatorname{Diff}(E, Q)$ and $\operatorname{Diff}\left(\bar{Q}^{*}, \bar{E}^{*}\right)$ of differential operators (105) and (106).

Proposition 17: Compositions of operators $v \circ v^{\prime}$ and $\Delta^{\prime} \circ \Delta$ obey the relations (3).

Proof: It suffices to prove the first relation. Let $v \circ v^{\prime} \in \operatorname{Diff}\left(E^{\prime}, Q\right)$ be a composition of differential operators $v \in \operatorname{Diff}(E, Q)$ and $v^{\prime} \in \operatorname{Diff}\left(E^{\prime}, E\right)$. Given fiber coordinates $\left(\xi^{r}\right)$ on $E \rightarrow Y,\left(\epsilon^{p}\right)$ on $E^{\prime} \rightarrow Y$ and $\left(\bar{q}_{a}\right)$ on $\bar{Q}^{*} \rightarrow Y$, this composition defines the density (109)

$$
\overline{v \circ v^{\prime}}=\sum_{\Lambda} v_{r}^{a, \Lambda} d_{\Lambda}\left(\sum_{\Sigma} v_{p}^{\prime r, \Sigma} \epsilon_{\Sigma}^{p}\right) \bar{q}_{a} \omega
$$

Following the relation (29), one can bring this density into the form

$$
\sum_{\Sigma} v_{p}^{\prime r, \Sigma} \epsilon_{\Sigma}^{p} \sum_{\Lambda}(-1)^{|\Lambda|} d_{\Lambda}\left(v_{r}^{a, \Lambda} \bar{q}_{a}\right) \omega+d_{H} \sigma=\sum_{\Sigma} v_{p}^{\prime r, \Sigma} \epsilon_{\Sigma}^{p} \sum_{\Lambda} \eta(v)_{r}^{a, \Lambda} \bar{q}_{\Lambda a} \omega+d_{H} \sigma
$$


Its Euler-Lagrange operator projected to $V_{Y}^{*} E^{\prime} \underset{E^{\prime}}{\otimes} T^{*} X$ is

$$
\sum_{\Sigma}(-1)^{|\Sigma|} d_{\Sigma}\left(v_{p}^{\prime r, \Sigma} \sum_{\Lambda} \eta(v)_{r}^{a, \Lambda} \bar{q}_{\Lambda a}\right) \bar{d} \epsilon^{p} \otimes \omega=\sum_{\Sigma} \eta\left(v^{\prime}\right)_{p}^{r, \Sigma} d_{\Sigma}\left(\sum_{\Lambda} \eta(v)_{r}^{a, \Lambda} \bar{q}_{\Lambda a}\right) \bar{d} \epsilon^{p} \otimes \omega
$$

that leads to the desired composition $\eta\left(v^{\prime}\right) \circ \eta(v)$.

\section{APPENDIX B}

The following is a graded counterpart of Theorem 16.

Let $T \rightarrow X$ and $W \rightarrow X$ be vector bundles, $W^{*}$ the dual of $W$, and $\bar{W}^{*}$ the densitydual (1) of $W$. Given the BGDA $\mathcal{S}_{\infty}^{*}[Q ; Y](53)$, let us consider its extensions to a BGDA $\mathcal{S}_{\infty}^{*}\left[T, W^{*}\right]$ with the local basis $\left\{s^{A}, t^{r}, w_{a}\right\}$, where elements $t^{r}$ and $w_{a}$ are either even or odd, and to a BGDA $\mathcal{S}_{\infty}^{*}\left[T, \bar{W}^{*}\right]$ possessing the local basis $\left\{s^{A}, t^{r}, \bar{w}_{a}\right\}$, where $\left[\bar{w}_{a}\right]=\left(\left[w_{a}\right]+\right.$ 1) $\bmod 2$.

Theorem 18: Given a graded function

$$
v=\sum_{0 \leq|\Lambda| \leq m} t_{\Lambda}^{r} v_{r}^{a, \Lambda}\left(x^{\lambda}, s_{\Sigma}^{A}\right) w_{a} \in \mathcal{S}_{\infty}^{0}\left[T, W^{*}\right]
$$

linear in $t_{\Lambda}^{r}$ and $w_{a}$, there exists a graded density

$$
\begin{aligned}
& \eta(v)=\sum_{0 \leq|\Lambda| \leq m} t^{r} \eta(v)_{r}^{a, \Lambda} \bar{w}_{\Lambda a} \omega \in \mathcal{S}_{\infty}^{0, n}\left[T, \bar{W}^{*}\right], \\
& \eta(v)_{r}^{a, \Lambda}=\sum_{0 \leq|\Sigma| \leq m-|\Lambda|}(-1)^{|\Sigma+\Lambda|} C_{|\Sigma+\Lambda|}^{|\Sigma|} d_{\Sigma}\left(v_{r}^{a, \Sigma+\Lambda}\right),
\end{aligned}
$$

linear in $t^{r}$ and $\bar{w}_{\Lambda a}$. Conversely, such a density

$$
\Delta=\sum_{0 \leq|\Lambda| \leq m} t^{r} \Delta_{r}^{a, \Lambda} \bar{w}_{\Lambda a} \omega
$$

defines the graded function

$$
\begin{aligned}
& \eta(\Delta)=\sum_{0 \leq|\Lambda| \leq m} t_{\Lambda}^{r} \eta(\Delta)_{r}^{a, \Lambda} w_{a} \in \mathcal{S}_{\infty}^{0}\left[T, W^{*}\right], \\
& \eta(\Delta)_{r}^{a, \Lambda}=\sum_{0 \leq|\Sigma| \leq m-|\Lambda|}(-1)^{|\Sigma+\Lambda|} C_{|\Sigma+\Lambda|}^{|\Sigma|} d_{\Sigma}\left(\Delta_{r}^{a, \Sigma+\Lambda}\right),
\end{aligned}
$$

linear in $t_{\Lambda}^{r}$ and $w_{a}$. 
Proof: The graded function $v$ (113) defines the graded density

$$
\bar{v}=\sum_{0 \leq|\Lambda| \leq m} t_{\Lambda}^{r} v_{r}^{a, \Lambda}\left(x^{\lambda}, s_{\Sigma}^{A}\right) \bar{w}_{a} \omega \in \mathcal{S}_{\infty}^{0}\left[T, \bar{W}^{*}\right]
$$

Its Euler-Lagrange operator $\delta(\bar{v})(60)$ contains the summand

$$
\mathcal{E}_{r} \theta^{r} \wedge \omega=\sum_{0 \leq|\Lambda| \leq m}(-1)^{|\Lambda|} \theta^{r} \wedge d_{\Lambda}\left(v_{r}^{a, \Lambda} \bar{w}_{a}\right) \omega
$$

which defines the graded density

$$
\sum_{0 \leq|\Lambda| \leq m}(-1)^{|\Lambda|} t^{r} d_{\Lambda}\left(v_{r}^{a, \Lambda} \bar{w}_{a}\right) \omega
$$

owing to the canonical isomorphism $V^{*} T=T^{*} \times T$. Using the relation (30), we come to the formula (114). The converse is proved similarly to the proof of Theorem 16.

1 G.Barnich, F.Brandt and M.Henneaux, Local BRST cohomology in gauge theories, Phys. Rep. 338, 439 (2000).

2 R.Fulp, T.Lada and J.Stasheff, Noether variational Theorem II and the BV formalism, Rend. Circ. Mat. Palermo (2) Suppl. No. 71, 115 (2003).

3 G.Giachetta, L.Mangiarotti and G.Sardanashvily, Iterated BRST cohomology, Lett. Math. Phys. 53, 143 (2000).

4 G.Giachetta, L.Mangiarotti and G.Sardanashvily, Lagrangian supersymmetries depending on derivatives. Global analysis, Commun. Math. Phys. (accepted); E-print arXiv: hep-th/0407185.

5 G.Sardanashvily, Cohomology of the variational complex in field-antifield BRST theory, Mod. Phys. Lett. A 16 (2001) 1531.

6 D.Hernández Ruipérez and J.Muñoz Masqué, Global variational calculus on graded manifolds, J. Math. Pures Appl. 63, 283 (1984).

7 F.Brandt, Jet coordinates for local BRST cohomology, Lett. Math. Phys. 55, 149 (2001). 
${ }^{8}$ F.Takens, A global version of the inverse problem of the calculus of variations, J. Diff. Geom. 14, 543 (1979).

9 I.Anderson, Introduction to the variational bicomplex, Contemp. Math. 132, 51 (1992).

10 G.Giachetta, L.Mangiarotti and G.Sardanashvily, Cohomology of the infinite-order jet space and the inverse problem, J. Math. Phys. 424272 (2001).

11 I.Anderson and T.Duchamp, On the existence of global variational principles, Amer. J. Math. 102, 781 (1980).

12 L.Mangiarotti and G.Sardanashvily, Connections in Classical and Quantum Field Theory (World Scientific, Singapore, 2000).

13 L.Fatibene, M.Ferraris, M.Francaviglia and R.McLenaghan, Generalized symmetries in mechanics and field theories, J. Math.. Phys. 43, 3147 (2002).

${ }^{14}$ G.Giachetta, L.Mangiarotti and G.Sardanashvily, Noether conservation laws in higherdimensional Chern-Simons theory, Mod. Phys. Lett. A 18, 2645 (2003).

15 M.Fish and M.Henneaux, Homological perturbation theory and algebraic structure of the antifield-antibracket formalism for gauge theories, Commun. Math. Phys. 128, 627 (1990).

16 C.Bartocci, U.Bruzzo and D.Hernández Ruipérez, The Geometry of Supermanifolds (Kluwer, Dordrecht, 1991).

17 G.Sardanashvily, On algebras of gauge transformations in a general setting, E-print arXiv: math.QA/0411635. 